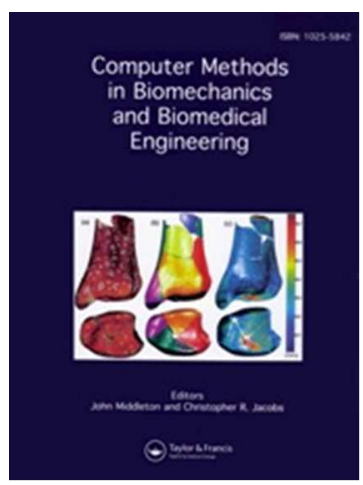

\title{
Development of a geometrically accurate and adaptable finite element head model for impact simulation: the Naval Research Laboratory-Simpleware Head Model
}

\begin{tabular}{|r|l|}
\hline Journal: & Computer Methods in Biomechanics and Biomedical Engineering \\
\hline Manuscript ID: & Draft \\
\hline Manuscript Type: & Original Article \\
\hline Complete List of Authors: & $\begin{array}{l}\text { Cotton, Ross; Simpleware Ltd., } \\
\text { Pearce, Christopher; Simpleware Ltd., } \\
\text { Young, Philippe; University of Exeter, College of Engineering, Mathematics } \\
\text { and Physical Sciences } \\
\text { Kota, Nithyanand; Leidos Corporation, MCET Department } \\
\text { Leung, Alan; US Naval Research Laboratory, Multifunctional Materials } \\
\text { Branch } \\
\text { Bagchi, Amit; US Naval Research Laboratory, Multifunctional Materials } \\
\text { Branch } \\
\text { Qidwai, Siddiq; US Naval Research Laboratory, Multifunctional Materials } \\
\text { Branch }\end{array}$ \\
\hline Keywords: & $\begin{array}{l}\text { head impact, blast, traumatic brain injury (TBI), simulation, finite element, } \\
\text { meshing }\end{array}$ \\
\hline & \\
\hline \multicolumn{2}{|c}{} \\
\hline
\end{tabular}

\section{SCHOLARONE \\ Manuscripts}


Development of a geometrically accurate and adaptable finite element head model for impact simulation: the Naval Research Laboratory-Simpleware

\section{Head Model}

R.T. Cotton, C.W. Pearce, P.G. Young, N. Kota, A.C. Leung, A. Bagchi, and S.M. Qidwai

Author names and information

Ross T. Cotton*, Christopher W. Pearce

Simpleware Ltd., Exeter, Devon, UK

Philippe G. Young

College of Engineering, Mathematics and Physical Sciences, University of Exeter, Exeter, Devon, $U K$

Nithyanand Kota

MCET Department, Leidos Corporation, Arlington, Virginia, USA

Alan Leung, Amit Bagchi, and Siddiq Qidwai

Multifunctional Materials Branch, US Naval Research Laboratory, Washington, USA

*Corresponding author

R.T. Cotton

E-mail: r.cotton@simpleware.com

Simpleware Ltd., Bradninch Hall, Castle Street, Exeter, EX4 3PL, UK

Research location

Research was conducted at Simpleware Ltd. and at the US Naval Research Laboratory. 


\section{Funding}

This work was supported by the Office of Naval Research (ONR) through the US Naval

Research Laboratory's Basic Research Program; and the Department of Defense (DoD) High

Performance Computing Modernization Program (HPCMP) using the Air Force Research

Laboratory (AFRL) Major Shared Resource Center (MSRC) under project 416, subproject 231. 


\title{
Development of a geometrically accurate and adaptable finite element head model for impact simulation: the Naval Research Laboratory-Simpleware
}

\section{Head Model}

\begin{abstract}
This study demonstrates a novel model generation methodology that addresses several limitations of conventional finite element head models. By operating chiefly in imagespace, new structures can be incorporated or merged, and the mesh either decimated or refined both locally and globally. This methodology is employed in the development of a highly bio-fidelic finite element head model from high resolution scan data. The model is adaptable and presented here in a form optimised for impact and blast simulations. The accuracy and feasibility of the model are successfully demonstrated against a widely used experimental benchmark in impact loading and through the investigation of potential brain injury under blast overpressure loading.
\end{abstract}

Keywords: head impact; blast; traumatic brain injury (TBI); simulation; finite element; meshing

\section{Introduction}

Traumatic brain injury (TBI) is a common pathology in both civil and warfare settings. In the United States there are approximately 1.7 million cases of TBI per year, resulting in around 52,000 deaths (US Centre for Disease Control and Prevention 2010). In the recent Iraq and Afghanistan conflicts, between $14-20 \%$ of surviving casualties have sustained a TBI (US Department of Veterans Affairs), and many are left with persistent neurological and motor function disorders (Schneiderman et al. 2008). Considerable research has been devoted to investigating the mechanisms which generate TBI in cases where the head is subjected to impact or blast. Of the various experimental methods available (in vivo human experiments, or tests on cadaveric, animal, physical, or mathematical models) the increasing availability of computing power has seen numerical simulation, and in particular the finite element (FE) method, come to the forefront of this research. The current study details the development of a 
highly realistic model of the whole human head and neck for use in numerical TBI related investigations.

\subsection{Finite element modelling}

FE simulation is an invaluable tool in the exploration of complex trauma mechanisms resulting from dynamic insults such as impacts and blasts. While sophisticated FE models of the head offer the prospect of providing accurate and repeatable experimental analyses of all categories of mechanical head injury, the validity of these models is heavily influenced by the geometric accuracy of the structures within the model. Traditionally computational models are created manually using computer aided design (CAD) tools, but when concerned with the complex anatomy of the head and its internal structures this manual approach has significant disadvantages; there is a large scope for subjectivity and user error, as well as of becoming rapidly computationally intractable as more geometric fidelity is introduced. A novel model generation approach which can reduce the inaccuracies associated with modelling a highly complex structure is known as 'image based meshing'. This refers to the conversion of volume scan data, generally from computed tomography (CT) or magnetic resonance imaging (MRI), directly into an FE mesh by way of fully- and semi-automated processes with minimal user input. This increases not only the accuracy, but also the speed at which computational models of complex geometries can be constructed, thereby allowing a greater number of anatomical features to be distinguished.

In 1997, Mehta et al. selected 14 cross-sectional image 'slices' from an MRI scan of the head and highlighted the skull in these images using an image processing tool. These highlighted outlines of the bone could then be read by a C++ computer code and converted into CAD coordinate and spline data, from which a model could be constructed that was, at least partially, based on the true anatomy of the skull (Mehta et al. 1997). Since early 
pioneering research such as this, image based modelling technology has developed dramatically. Many of the latest generation of sophisticated computational head models rely on image based meshing to a greater or lesser degree. The latest version of the Simulated Injury Monitor (SIMon) finite element head model (FEHM) developed at the US National Highway Traffic Safety Administration is based on CT data of the average adult male, and has been validated against many experimental measures; however, its primary application is for the assessment of the injury potential of recorded head kinematic data (from crash test dummies) (Takhounts et al. 2008). A recent study by Kraft et al. (2012) presents a complex image based FEHM in which the model's geometry was developed from T1 weighted MRI scan data, while diffusion tensor imaging (DTI) was used to inform an anisotropic material model of the connective neural tissue, to better represent material anisotropy of axonal structures throughout the brain (Kraft et al. 2012). Also of note are the Kungliga Tekniska Högskolan (KTH) model (Kleiven \& Von Holst 2002; Kleiven 2006), the University College Dublin Brain Trauma Model (UCDBTM) (Horgan \& Gilchrist 2004), the University of Salerno head model (El Sayed et al. 2008), and the University of Illinois head model (Chen \& Ostoja-Starzewski 2010), which are all examples of recent, highly detailed, image based FEHMs.

An issue with the extant FEHMs listed above is that they are, without exception, provided as 'fixed' or 'immutable' FE meshes due to the challenge in meshing from complex image data. Hence, modifications such as mesh refinement in areas of interest, incorporation of additional soft and hard tissue structures, or introduction of interacting components (helmets, probes, etc.) is challenging, if not impossible, as these changes must be carried out directly on the meshes. The consequence of this is that the models are rarely ideally suited for a given problem, and meshes quickly become outdated as computational resources and imaging techniques open up the prospect of more accurate models.

The present authors propose a new paradigm for providing head models which allows the end-user far more flexibility in adapting the model to the problem at hand and to the 
computational resources available. Rather than pre-defined meshes, the model exists as presegmented image data which can be both modified and then meshed based on problem requirements 'on the fly' by leveraging robust image based modelling techniques developed recently (Young et al. 2008).

\section{Methodology}

\subsection{Head model development}

\subsubsection{Data acquisition}

A high resolution whole head MRI scan of a male volunteer was obtained in vivo at the Royal Devon and Exeter Hospital, UK. The T1 weighted scan was taken axially, from the top of the head to its base; image 'slices' in the coronal plane had a resolution of $1.03516 \mathrm{~mm} \times$ $1.03516 \mathrm{~mm}$, with slice-to-slice separation of $1.04001 \mathrm{~mm}$. The volunteer was 25 years of age. His height and weight were measured immediately before the scan and were recorded as $1.8 \mathrm{~m}$ and $81 \mathrm{~kg}$ respectively; these measurements are close (less than $1.5 \%$ difference in each case) to the American $50^{\text {th }}$ percentile male (NASA 1995). Figure 1 depicts a section view of the head obtained from this MRI data. 


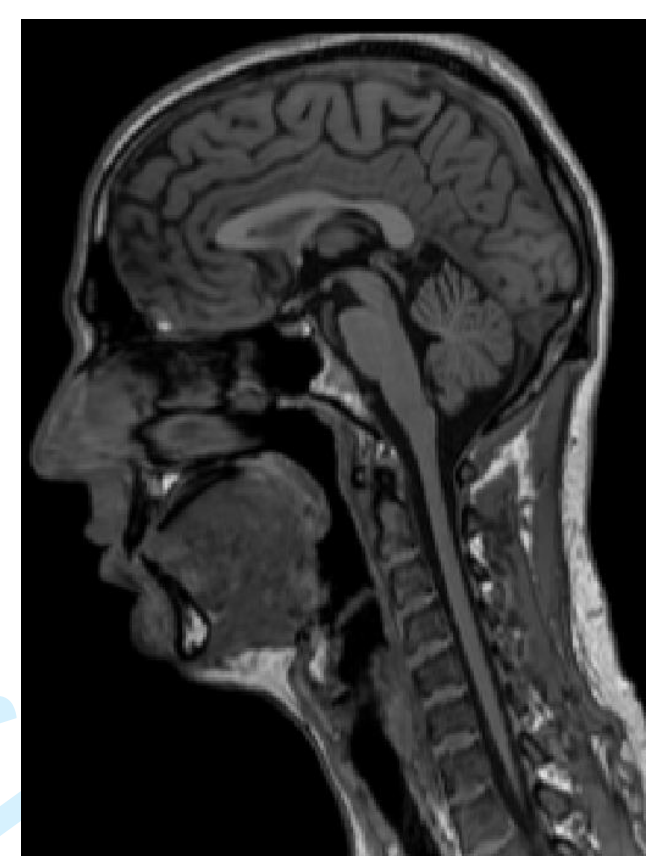

Figure 1: MRI image data used in the development of this model: sagittal section view of the head and interior structures.

\subsubsection{Image segmentation}

The process of segmentation entails the identification and labelling of regions of interest (e.g. brain, skull, muscles) within the greyscale data, creating so-called 'masks', which eventually form the basis for surface reconstruction and volume meshing. The masks representing different structures consist of flagged 'voxels' (i.e. the three dimensional equivalent of a pixel) in the image data. The DICOM (Digital Imaging and Communications in Medicine) image series obtained from the MRI scanner was imported directly into the image processing software ScanIP ${ }^{\mathrm{TM}}$ (Simpleware Ltd.) and a range of techniques were employed in order to build the masks of soft and hard tissue structures. Image threshold and flood-fill algorithms were used extensively to build the initial geometries of the major structures of the head. Where neighbouring regions had a similar greyscale value, manual segmentation allowed the structures to be disconnected. These steps were used to create such parts as the cerebellum (separate white and grey matter), cerebrum (separate white and grey matter) and 
brain stem. Image filters were also used on the masks in order to smooth these regions (Recursive Gaussian, median, and mean filters), edit the morphology or fill cavities (dilate, erode, open, and close functions). Finally, Boolean operations were applied to eliminate any overlapping masks in preparation for meshing.

\subsubsection{Image enrichment and modification: introducing additional structures}

In addition to the segmentation techniques mentioned above, for regions where the greyscale contrast was low such that it was impossible to differentiate certain structures, the image was enriched by incorporating CAD models of these structures. The facial and neck muscles (Figure 2(a)) were imported from a library of STL (Standard Tessellation Language) surface triangulations of human anatomical parts. These were scaled and positioned appropriately, and then converted to image data through a process known as 'voxelisaton', wherein a distance function is used to calculate representative greyscale information, such that they could be incorporated into the original scan image.

(a)

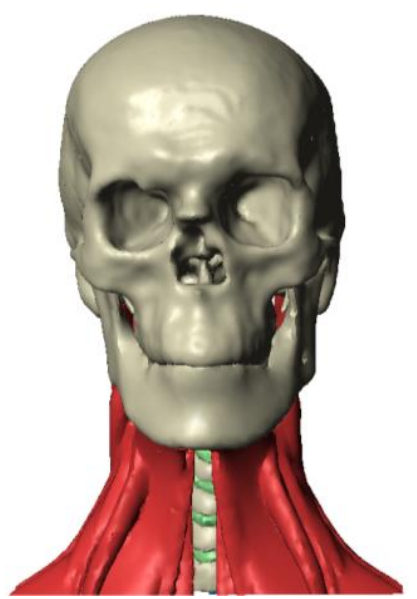

(b)

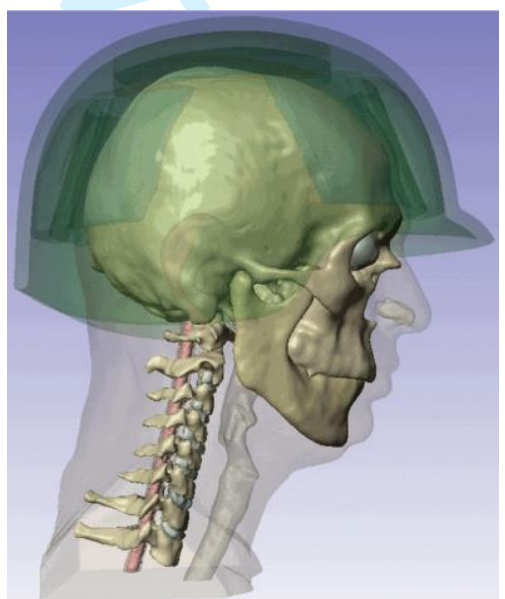

Figure 2: Image enrichment: (a) introduction of neck muscles from external CAD source, (b) an example of a possible study specific modification - incorporation of a helmet design.

It should be stressed here that the ability to straightforwardly modify the segmented data used as a basis for the mesh generation is a key strength of the proposed image based modelling 
paradigm. Modifications to include a helmet (Figure 2(b)), neural probes, introduce pathologies, or conflagrate different structures into one are just some of the examples of adaptations that one could perform to adjust the model to suit different studies.

The full list of anatomical structures differentiated in the current version of the head model is provided below in Table 1, and depicted in Figure 3.

\begin{tabular}{llrlll}
\hline & & Structures differentiated, defined as separate element sets \\
1 & Skin & 12 & Cerebrum - grey matter & 23 & Spinal cord \\
2 & Muscles & 13 & Cerebellum - grey matter & 24 & Ventricles - lateral (right) \\
3 & Soft tissues & 14 & Cerebrum - white matter & 25 & Ventricles - lateral (left) \\
4 & Skull inner and outer table & 15 & Cerebellum - white matter & 26 & Ventricles - third \\
5 & Skull diploë & 16 & Falx cerebri & 27 & Ventricles -fourth \\
6 & Mandible & 17 & Tentorium cerebelli & 28 & Ventricles - Aqueduct of Sylvius \\
7 & Cervical vertebrae & 18 & Eyes & 29 & Ventricles - Foramen of Monro \\
8 & Intervertebral discs & 19 & Optic nerves & 30 & Venous sinuses and bridging veins \\
9 & CSF & 20 & Brain stem - medulla & 31 & Venous sinuses and bridging veins \\
10 & Frontal sinus & 21 & Brain stem - midbrain & 32 & Dura mater (shell elements) \\
11 & Maxillary sinus & 22 & Brain stem - pons & 33 & Sclera (eyes) (shell elements) \\
\hline
\end{tabular}

Table 1: Full list of anatomical structures in current head model. Anatomical structures are numbered and labelled in Figure 3. 
(a)
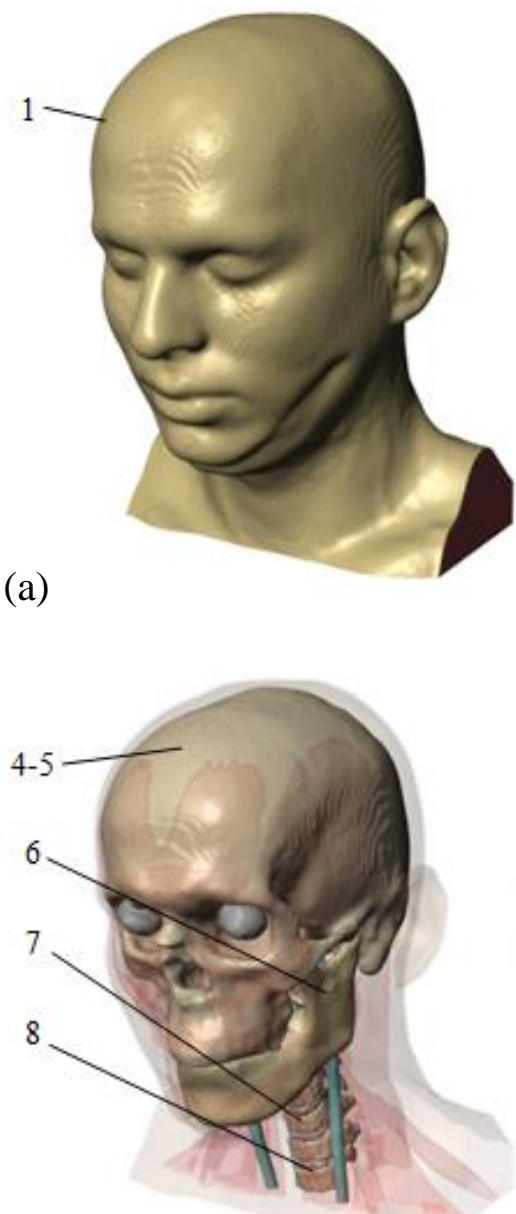

(d)

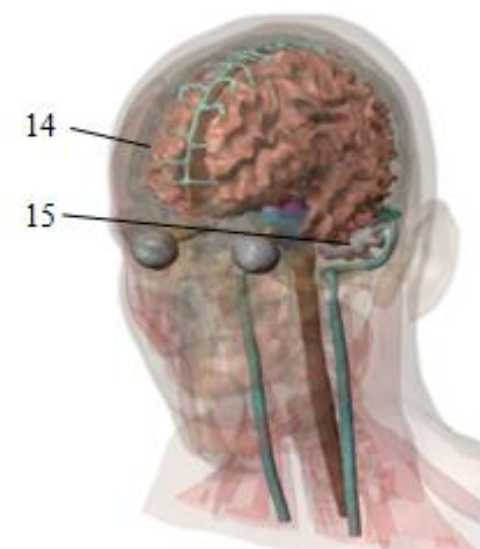

(g)

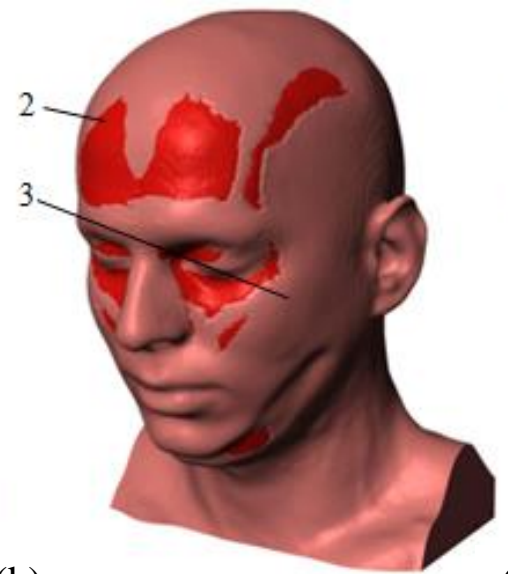

(b)

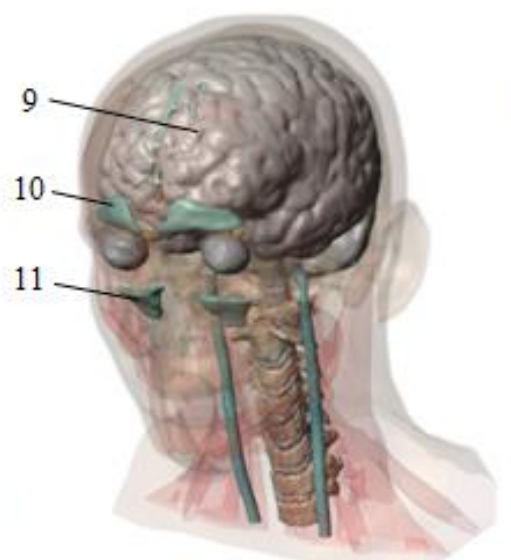

(e)

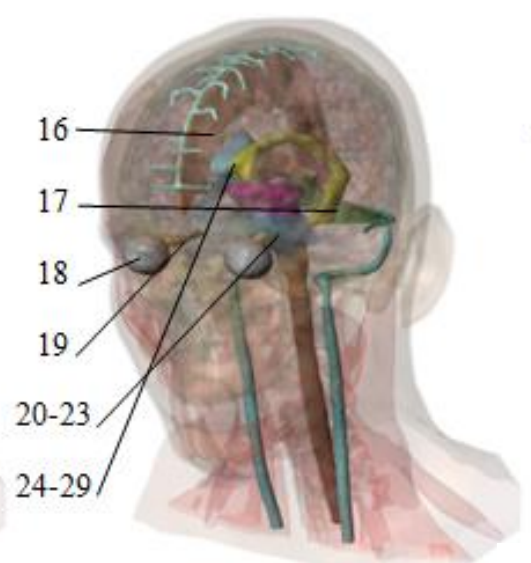

(h)

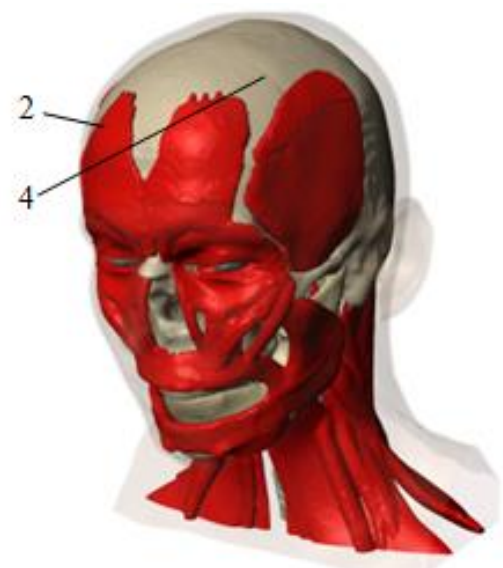

(c)

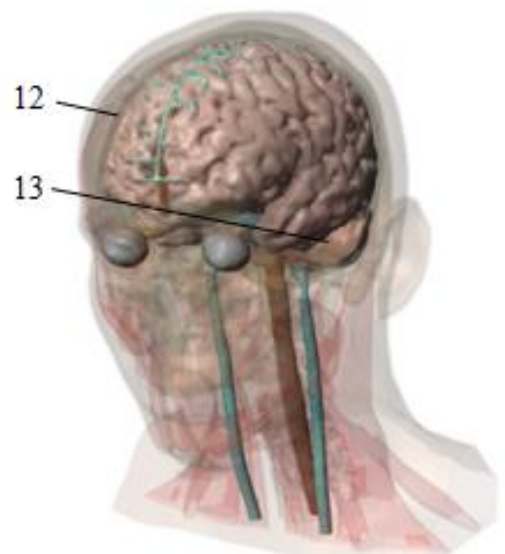

(f)

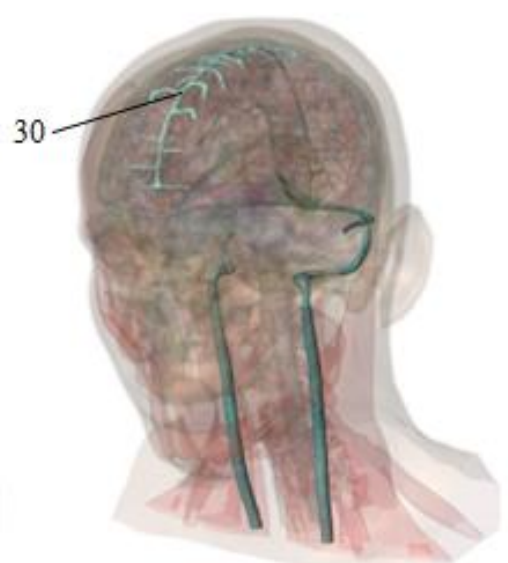

(i)

Figure 3: Complete model: (a) outer skin, (b) soft tissues and muscles, (c) muscles and skull, (d) skull, (e) CSF and sinuses, (f) cerebrum grey matter, (g) cerebrum white matter, (h) tentorium cerebelli, falx, and ventricles, and (i) venous sinuses. 


\subsubsection{Image based mesh generation}

Once a dataset has been segmented into 'regions of interest' (ROIs), the meshing process can broadly take one of two routes: (1) the ROIs are converted directly into volumetric FE models using a multi-part Extended Volumetric Marching Cubes (EVOMAC) approach (Young et al. 2008) or, alternatively; (2) the boundaries of the voxelised ROIs are converted into surface representations (e.g. triangulated surfaces such as STL, or NURBS surfaces) which can then be used as a basis for mesh generation using a range of automated mesh generation algorithms. The ideal method is dependent on the complexity of the geometry and desired number of elements in the final mesh. An overview of this process is presented below in Figure 4. Here both approaches are demonstrated using the $+\mathrm{FE}^{\mathrm{TM}}$ meshing software (Simpleware Ltd.).

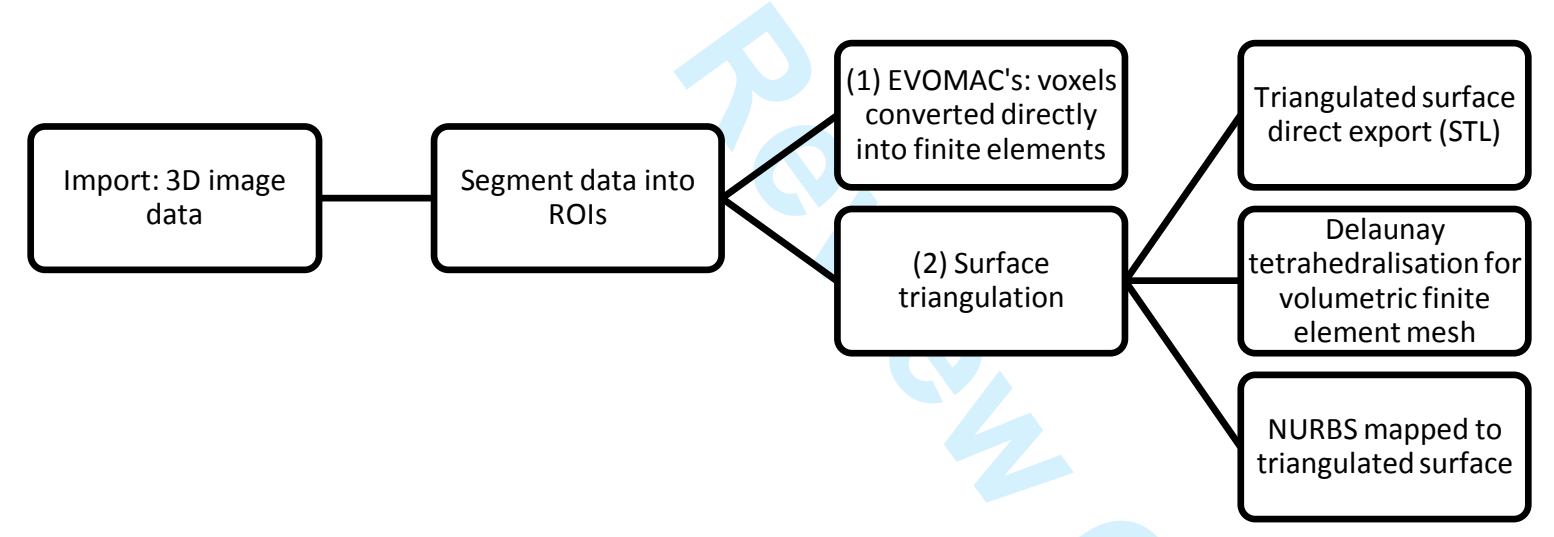

Figure 4: General overview of model generation procedure following image acquisition: various routes exist according to desired meshing operation and export type.

Mesh generation was carried out using both routes (1) and (2), i.e. with an EVOMAC-based mesher, and with a multi-part surface decimation algorithm followed by mixed Delaunay advancing front approach (Young et al. 2008). The EVOMAC-based meshing approach produces a structured mesh composed of both hexahedral and tetrahedral elements (see Figure 6(a)) or purely of tetrahedral elements. In the second approach, surface triangulation followed by Delaunay tetrahedralisation, produced unstructured, fully tetrahedral meshes (see 
Figure 5 and Figure 6(b)); the advantage of this approach being that it provides far more flexibility in terms of controlling element sizes and the final element count. The model shown in Figure 5 and Figure 6(b) was developed as part of an ongoing collaborative project between the United States Naval Research Laboratory and Simpleware Ltd.

Table 2, below, lists the final element count for each of these different mesh configurations, along with common mesh quality metrics, such as average element in-out aspect ratio and minimum edge length aspect ratio.

\begin{tabular}{lll}
\hline & \multicolumn{1}{c}{$\begin{array}{c}\text { Structured mixed hexahedral } \\
\text { and tetrahedral mesh }\end{array}$} & \multicolumn{1}{c}{ Unstructured Delaunay method } \\
& tetrahedral mesh \\
\hline Corresponding & Figure 6(a) & Figure 5 and Figure 6(b) \\
images & & \\
No. of elements & $22.81 \mathrm{M}$ & $3.72 \mathrm{M}$ \\
No. of nodes & $5.89 \mathrm{M}$ & 695,691 \\
Av. in-out & 0.683 & 0.683 \\
Min. in-out & 0.096 & 0.083 \\
Av. aspect ratio & 1.842 & 1.941 \\
Min. Aspect ratio & 13.148 & 12.523 \\
Min. dihedral angle & 5.352 & 7.910 \\
\hline
\end{tabular}

Table 2: Number of elements and nodes generated from two different mesh configurations using the structured and unstructured meshing algorithms, as well as metrics relating to the quality of these meshes. 

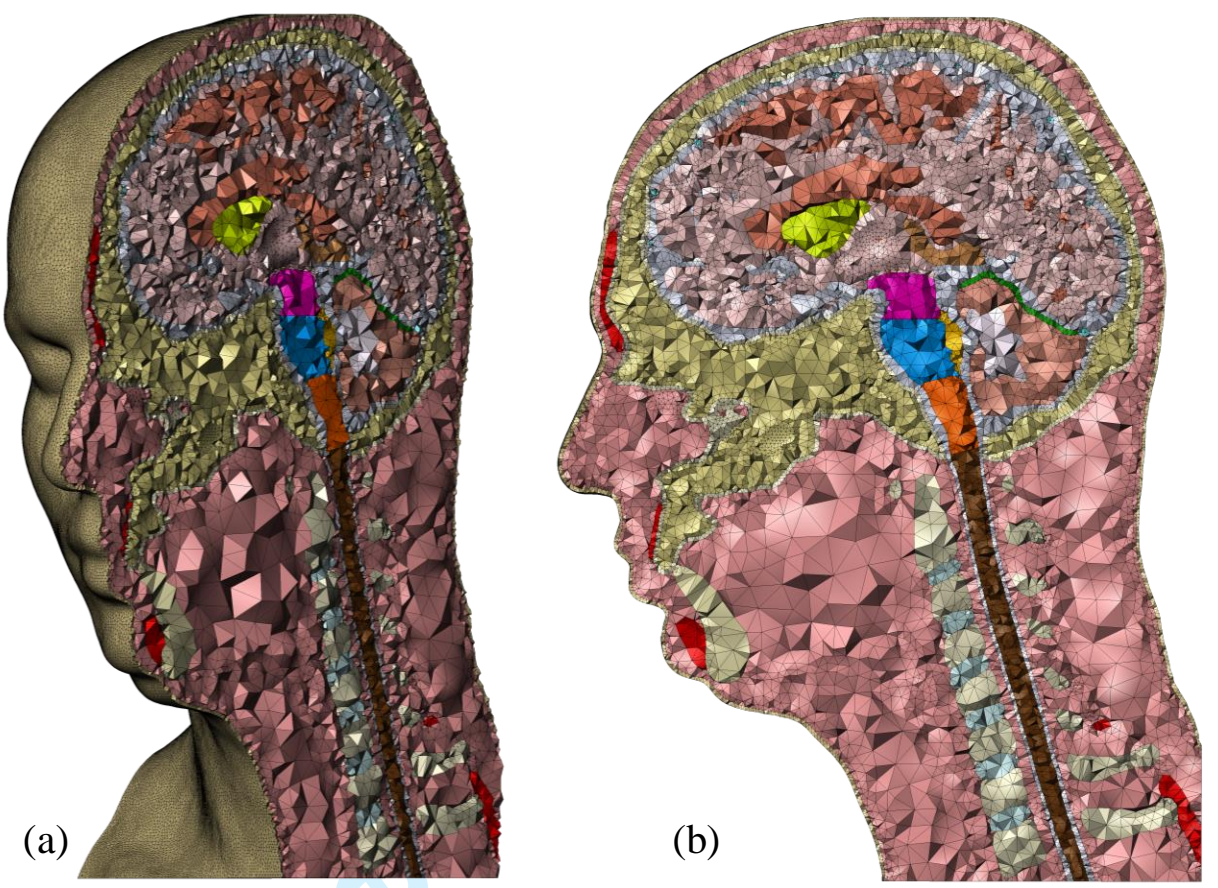

Figure 5: Sagittal section of the unstructured, all tetrahedral mesh with $3.72 \mathrm{M}$ volumetric elements: (a) oblique view, (b) normal view.

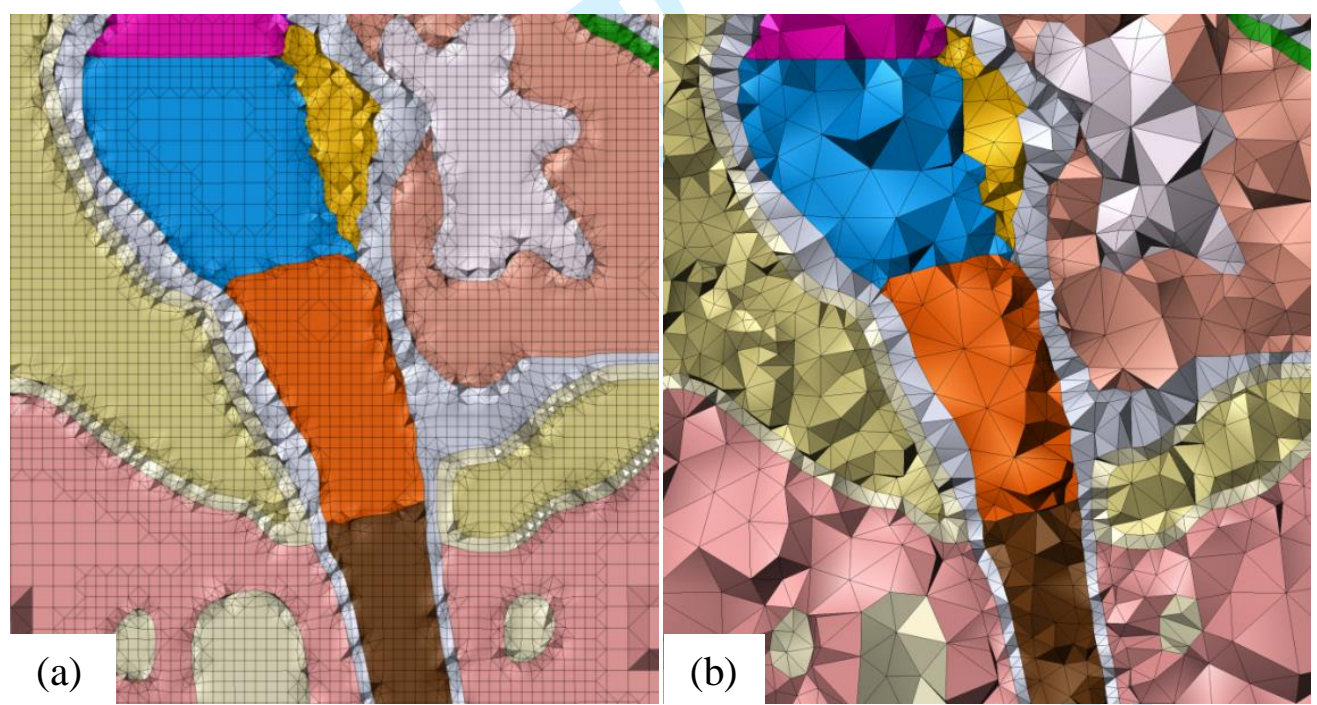

Figure 6: Close-up views of (a) structured mixed element mesh, and (b) unstructured tetrahedral mesh, highlight the ability of the unstructured approach to retain geometric detail while allowing reduced element numbers and hence computational cost.

\subsubsection{Additional mesh modifications}

Once the mesh has been generated, additional information can be added to make it simulation 
ready:

- Contact pairs can be specified to allow for sliding surfaces or separation.

- Node and surface sets can be added based on mask geometry or in arbitrary userdefined regions.

- If desired, material properties can be defined which are based on the underlying greyscale intensity of the image.

- Boolean operations applied directly to structures in image-space are efficient and robust compared to the application of these processes to existing mesh geometries. Features or specific regions can straightforwardly be merged or removed, thus reducing element count if a distinction is not needed. Figure 7 shows an example of how the brain can be merged into one domain.

(a)
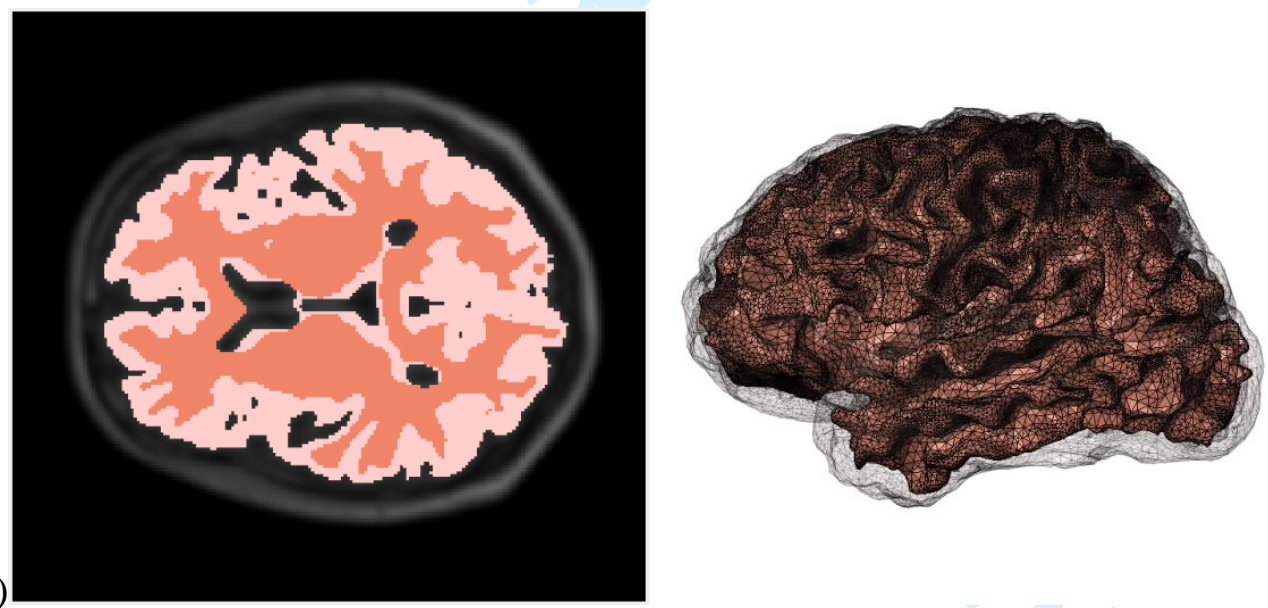
(b)
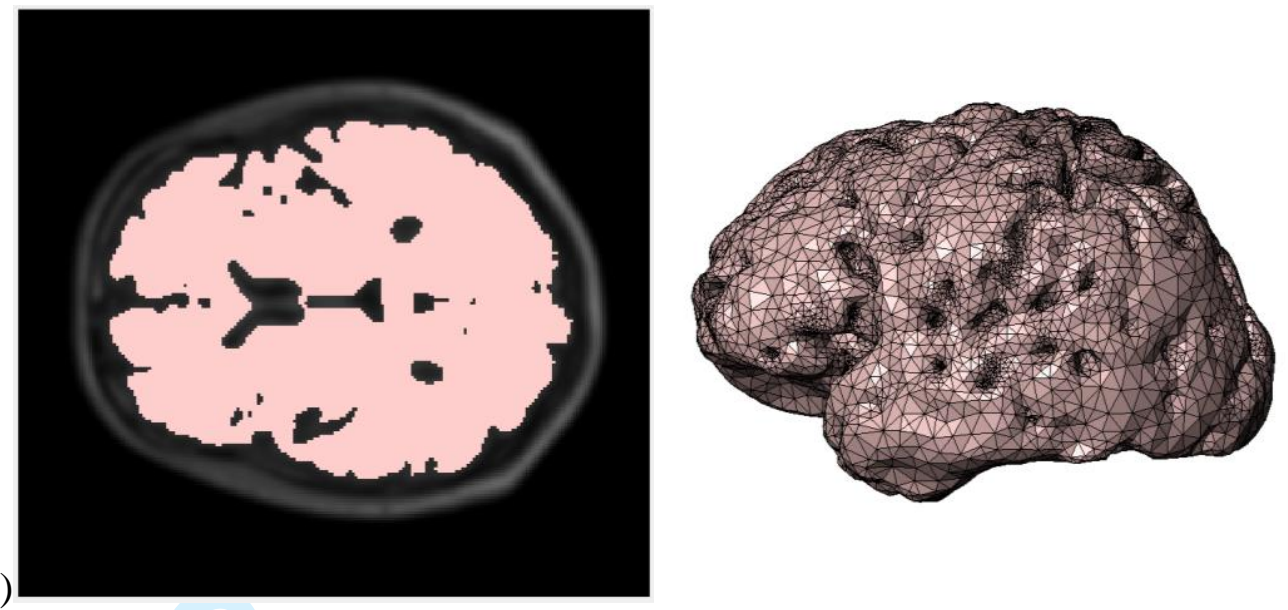

Figure 7: Using Boolean operations on the segmented masks to merge parts: (a) white and grey matter, (b) merged to one domain.

\subsection{Adaptation of NRL-Simpleware prototype model for simulations}

As mentioned earlier, the prototype NRL-Simpleware modelling scheme is sufficiently flexible to create tailor-made FE models. To simulate human head injury under mid- to highrate impact and blast loading conditions, a hybrid mesh was created by modifying the unstructured mesh shown in Figure 5. This tetrahedral mesh was initially composed of linear elements. While 4-node linear elements are computationally inexpensive, they also have a tendency to undergo volumetric locking (exhibiting the familiar checkerboard effect) when used to model near-incompressible materials like the brain under high rates of deformation.

On the other hand, the computationally more expensive 10-node quadratic tetrahedral elements avoid locking while allowing high-fidelity representation of geometry. For high-rate simulations, a compromise between computational efficiency and accuracy was obtained by building the mesh comprising regions of near-incompressible materials, such as the cerebrospinal fluid (CSF) and brain, with quadratic elements. To accommodate this arrangement, additional changes in the nodal definitions had to be performed to describe the interface of quadratic and linear parts of the FE mesh. These modifications included splitting shared nodes between quadratic and linear elements (to ensure no co-penetration of the 
additional nodes on the quadratic side into the linear elements), and defining appropriate contact interactions at such interfaces. The following list summarises these changes:

- Conversion of linear elements into quadratic elements in the intracranial regions of near-incompressible materials: brain, spinal cord, CSF, sinuses filled with air, ventricles filled with CSF, veins filled with fluid, and eyes.

- Creation of contact surfaces between: quadratic elements of veins and linear elements of skull, veins and soft tissue outside the intracranial region, and eyes and soft tissue/skin, through duplication of shared nodes at the interfaces. Tied contact conditions were imposed for these interfaces.

- Partitioning of skull and mandible into multiple regions with similar outward facing normal vectors to assign transversely isotropic material properties to each region.

Tied contact conditions were imposed between the sub-arachnoid region filled with CSF, and between the dura mater and pia mater, respectively. Wherever applicable, the same conditions were also imposed for the spinal cord. The material models for individual components were selected based on data from quasi-static to high-rate experiments whenever available. The functional forms of these models ranged from simpler linear elastic and equations of state to relatively more complex hyper-viscoelastic models. The calibration of the later models was performed with a modified Monte-Carlo based optimisation scheme. The type of constitutive model and the calibrated parameters for all head components are provided in Table A1 in the appendix. Similarly, thicknesses of the various membrane components in the head are given in Table A2. Details of constitutive models, calibrations schemes, and overall verification and validation of the computational model can be found in Kota et al. 2014. 
The adapted model was used for simulating both blunt impact (low-to-mid rate) and blast overpressure (mid-to-high rate) scenarios. The simulations were performed with the commercial FE software Abaqus/Explicit ${ }^{\mathrm{TM}}$ using 200 Intel $^{(\mathrm{R})}$ cores (2GB RAM/core; 2.6 GHz core speed) on an SGI Ice X system. A variable mass scaling scheme was utilised to ensure a reasonable time increment of $50 \mathrm{nsec}$ or more, which allowed completion of approximately $20 \mathrm{msec}$ of total simulation time in 24 hours. The resulting change of total model mass was monitored during simulations and was observed to be lower than $0.09 \%$ throughout, indicating no significant effect on the inertial characteristics.

\section{Applications}

\subsection{Blunt impact loading}

In this case, a blunt impact experiment performed on a Post-Mortem Human Subject (PMHS) by Nahum et al. 1977 to reproduce automotive impact conditions is modelled computationally. The experiment (\#37 in the original study) involved frontal impact to the head at 45 degrees by a padded impactor of $5.6 \mathrm{~kg}$ mass travelling at $9.94 \mathrm{~m} / \mathrm{s}$ (Figure 8 ). The simulation was performed using the experimental force recorded on the head as input to the model. No other boundary conditions were applied. The predicted intracranial pressures are compared with experimental data for frontal and parietal regions in Figure 9(a) and Figure 9(b), respectively. The simulated pressures reasonably capture the pressure histories recorded inside the skull. 

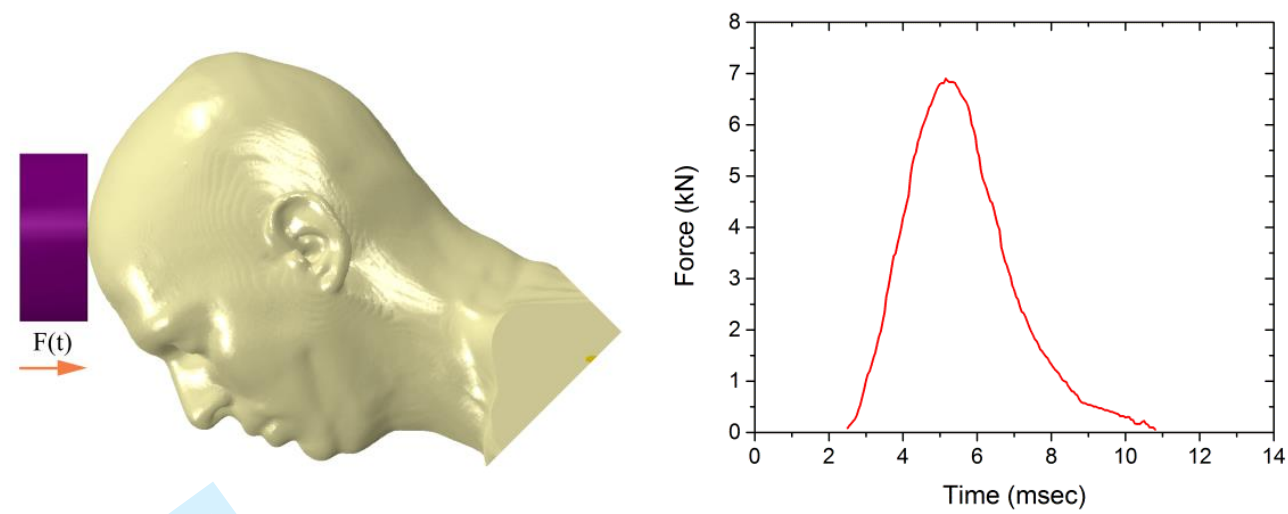

Figure 8: (Left) Schematic of a padded impactor hitting the head model at 45 degree angle; (Right) Instead of the impactor, the experimental force-time plot was used in this study.

The main advantage of computational modelling is the accruement of detailed spatial data that is otherwise usually not practical to collect from experiments. For example, postprocessing of the simulation data can be performed to quantify the injured brain volume as a function of event time based on injury threshold measures described in the literature for focal and diffuse injuries (Bešenski 2002). For the present simulation, Python scripting was used to calculate the evolution of these volumes from the large output database. As an example, the temporal history for focal injury volume based on pressure threshold of $173 \mathrm{KPa}$ (Zhang et al. 2004) and diffuse injury volume based on shear strain threshold of 5\% (Margulies et al. 1992) are shown in Figure 9(c). These post processing capabilities highlight the utility of such computational models.
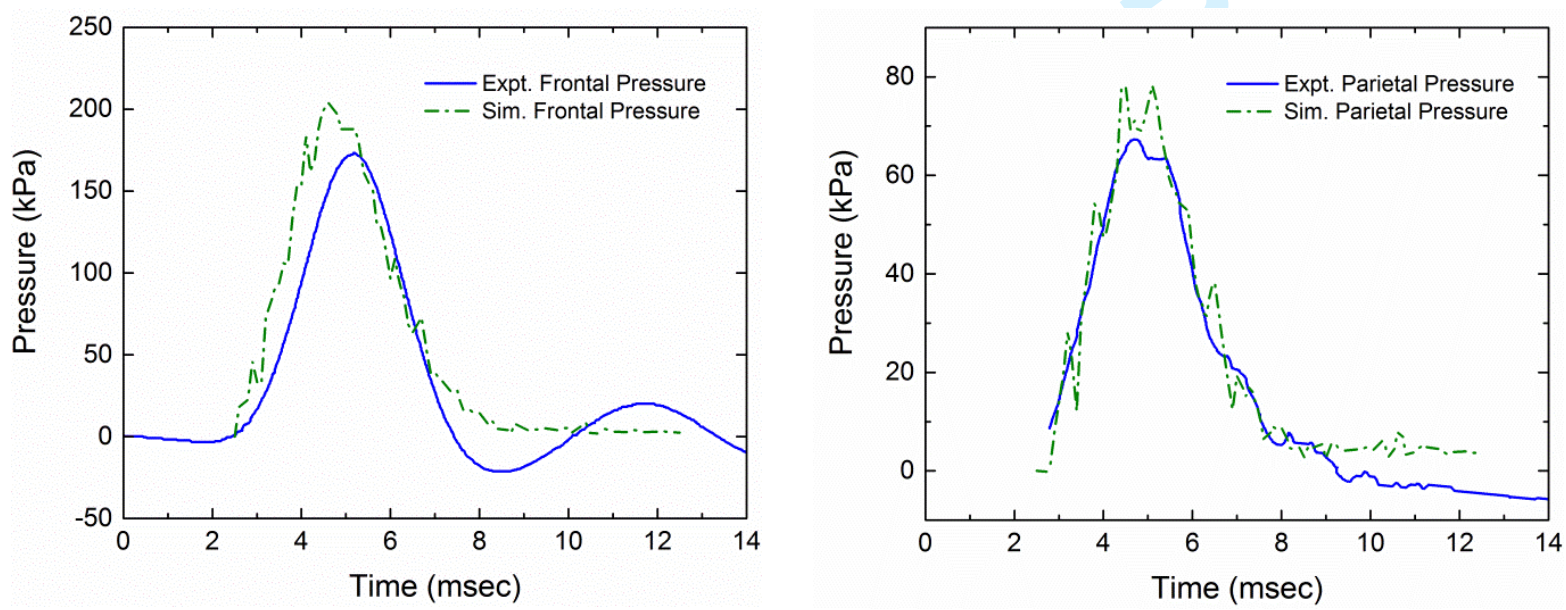


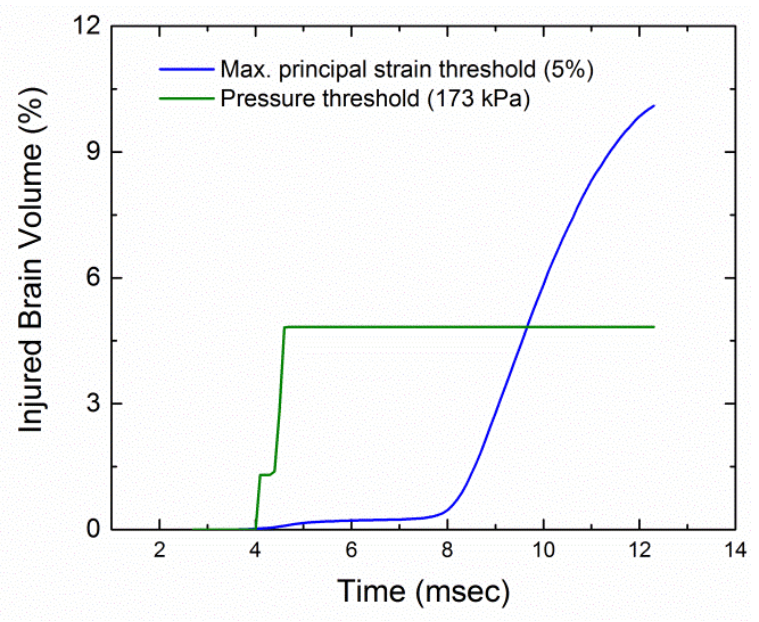

(c)

Figure 9: Comparison between experimental and predicted pressures for blunt impact to the head in (a) frontal and (b) parietal regions of the brain, respectively; (c) Temporal history of injured brain volume based on maximum principal strain threshold of 5\% and pressure threshold of $173 \mathrm{kPa}$.

\subsection{Blast overpressure loading}

The blast overpressure loading was simulated by choosing the incident wave loading option in Abaqus/Explicit. In this option, the magnitude of the applied pressure pulse is specified on a plane with a specific normal vector. As the wave progresses in the direction of the normal, it encounters the target in a manner akin to a true overpressure impact; however, it does not allow wraparound effect. In the current work, a frontal loading with a Friedlander wave profile was chosen; the peak pressure was approximately $430 \mathrm{kPa}$ (Figure 10).

The passage of the pressure wave passing across the face and head is shown in Figure 11. The injurious effect of this pressure pulse can be displayed in a temporal-spatial form by collecting the temporal injury data for each individual material point and plotting it over the target volume. Such graphics can then be used to predict injury patterns or draw spatial comparisons among different metrics of injury to determine their relative efficacy in 
predicting actual injury. For example, brain regions based on thresholds of $11 \mathrm{kPa}$ effective stress (Kang et al. 1997) for focal injury and 5\% maximum principal strain (Margulies et al. 1992) for diffuse injury, respectively, are highlighted in Figure 12(a) and (b). The red coloured regions represent cumulative injury in the first 5 milliseconds based on each threshold.

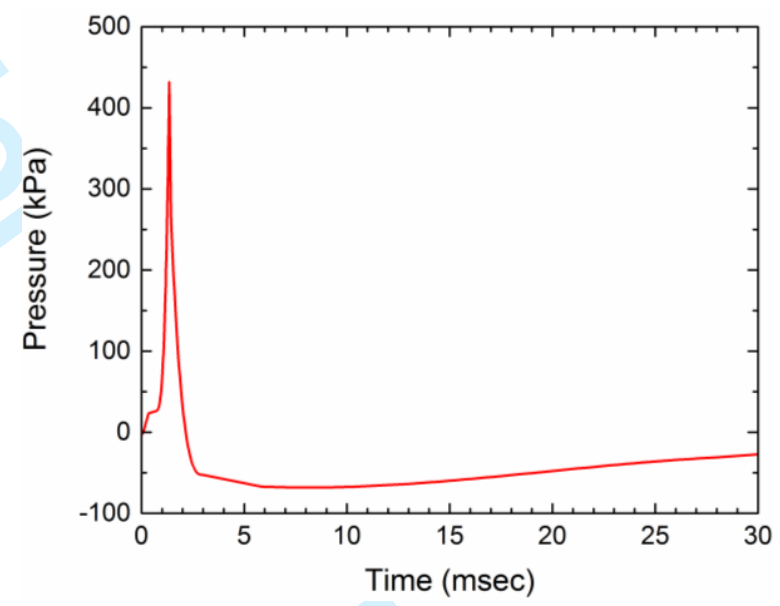

Figure 10: Friedlander wave profile used in frontal blast loading simulation.

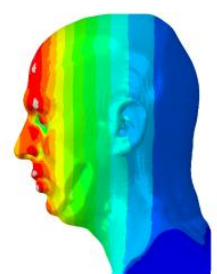

$1.4 \mathrm{msec}$

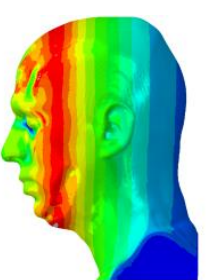

$1.5 \mathrm{msec}$

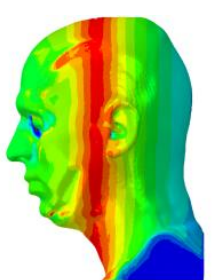

$1.6 \mathrm{msec}$

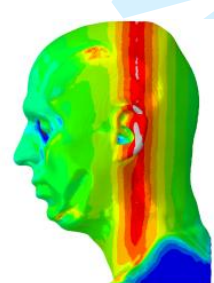

$1.7 \mathrm{msec}$

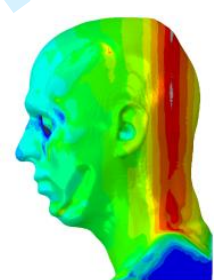

$1.8 \mathrm{msec}$

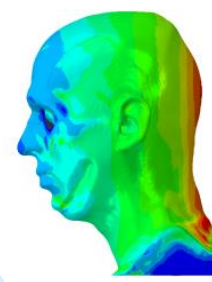

$1.9 \mathrm{msec}$
Pascals

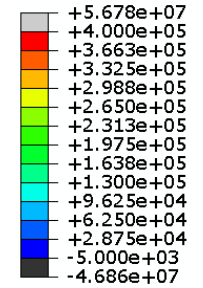

Figure 11: Passage of peak pressure pulse across the face during loading. 


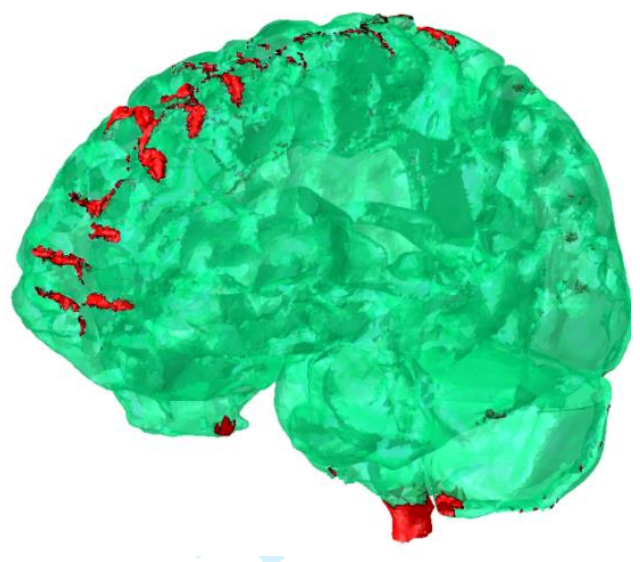

(a) Effective Stress Prediction

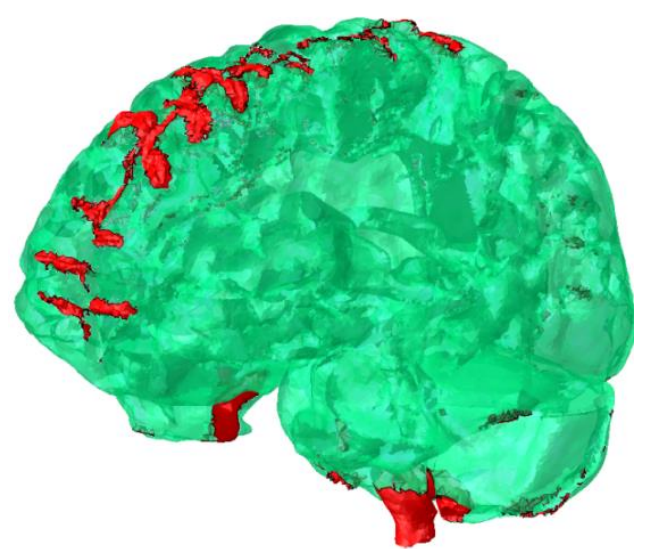

(b) Max. Principal Strain Prediction

Figure 12: Spatial identification of injured brain regions due to blast overpressure loading based on: (a) effective stress threshold of $11 \mathrm{kPa}$ for focal injury and (b) maximum principal strain threshold of 5\% for diffuse injury, up to $5 \mathrm{msec}$. The regions coloured red have experienced values beyond the respective thresholds.

\section{Conclusion}

The modelling approach demonstrated here is fundamentally distinct from the conventional manual creation of finite element models. By exploiting recent advances in image processing and meshing techniques, numerical head models can be developed that benefit from being extremely flexible; this methodology allows the resulting head models to be optimised for specific investigations.

Through the simulated recreation of Nahum et al's (1977) experiment, the highly biofidelic head model developed by the US Naval Research Laboratory and Simpleware Ltd. was successfully shown to be in excellent agreement with these benchmark results. In addition, the model was subjected to a blast overpressure simulation typical of military and improvised explosives weapons. The results were analysed in conjunction with both stress and strain based injury criteria obtained from the literature and they showed the capability of the model in predicting brain injury as a function of both location and time. 
The current approach is capable of capturing the minute details of the complex head and brain geometry in even greater detail than is presented here, but at present is limited by the number of elements that can be solved by current numerical solvers in a reasonable timeframe. Image based meshing has a promising future and is set to harness the continuous improvement of 3D imaging techniques to fulfil its potential to yield extremely bio-fidelic models of arbitrary complexity, enabled by exponential advances in computing power and parallelisation.

\section{References}

Bešenski N. Traumatic injuries: Imaging of head injuries. European Radiology 2002; 12(6): 1237-1252.

Chen Y, Ostoja-Starzewski M. 2010. MRI-based finite element modeling of head trauma: spherically focusing shear waves. Acta Mech. 213:155-167.

Horgan T, Gilchrist MD. 2004. Influence of FE model variability in predicting brain motion and intracranial pressure changes in head impact simulations. Int J Crashworthiness. 9:401-418.

Kang HS, Willinger R, Diaw BM and Chinn B. Validation of a 3D anatomic human head model and replication of head impact in motorcycle accident by finite element modeling. SAE transactions 1997; 106(6): 3849-3858.

Kleiven S, Von Holst H. 2002. Consequences of head size following trauma to the human head. J Biomech. 35:153-60.

Kota N, Bagchi A and Qidwai SM. On the challenges in verification and validation of biomechanical models of humans: A case study on head model development for impact loading. J Biomech 2014 Submitted.

Kleiven S. 2006. Evaluation of head injury criteria using a finite element model validated against experiments on localized brain motion, intracerebral acceleration, and intracranial pressure. Int J Crashworthiness. 11:65-79.

Kraft R, Mckee P, Dagro A, Grafton S. 2012. Combining the finite element method with structural connectome-based analysis for modeling neurotrauma: connectome neurotrauma mechanics. PLoS Comput Biol [Internet]. [cited 2013 Jul 31]; 8. Available from: http://dx.plos.org/10.1371/journal.pcbi.1002619 
Margulies SS and Thibault LE. A proposed tolerance criterion for diffuse axonal injury in man. Journal of Biomechanics 1992; 25(8):917-923.

Mehta B V, Rajani S, Sinha G. 1997. Comparison of image processing techniques (magnetic resonance imaging, computed tomography scan and ultrasound) for 3D modeling and analysis of the human bones. J Digit Imaging. 10:203-6.

Nahum AM, Smith R and Ward CC. Intracranial pressure dynamics during head impact. Stapp Car Crash Conference Proceedings 1977: 339-366.

NASA. 1995. Volume 1, Section 3: Anthropometry and biomechanics. Man-system Integr Stand [Internet]. [cited 2012 Nov 5]. Available from: http://msis.jsc.nasa.gov/sections/section03.htm

El Sayed T, Mota A, Fraternali F, Ortiz M. 2008. Biomechanics of traumatic brain injury. Comput Methods Appl Mech Eng. 197:4692-4701.

Schneiderman AI, Braver ER, Kang HK. 2008. Understanding sequelae of injury mechanisms and mild traumatic brain injury incurred during the conflicts in Iraq and Afghanistan: persistent postconcussive symptoms and posttraumatic stress disorder. Am J Epidemiol. 167:1446-52.

Simpleware Ltd. 2013. Reference Guide, ScanIP Software +FE, +NURBS, +CAD Modules. Simpleware Ltd, Bradninch Hall, Castle Street, Exeter, EX4 3PL, United Kingdom. Takhounts EG, Ridella S a, Hasija V, Tannous RE, Campbell JQ, Malone D, Danelson K, Stitzel J, Rowson S, Duma S. 2008. Investigation of traumatic brain injuries using the next generation of simulated injury monitor (SIMon) finite element head model. Stapp Car Crash J. 52:1-31.

US Centre for Disease Control and Prevention. 2010. Get the stats on traumatic brain injury in the United States [Internet]. [cited 2012 Jun 17]:2. Available from: http://www.cdc.gov/traumaticbraininjury/pdf/BlueBook_factsheet-a.pdf (updated 17th March 2010)

US Department of Veterans Affairs. Traumatic brain injury: a guide for patients [Internet]. Available from: http://www.mentalhealth.va.gov/docs/tbi.pdf Young PG, Beresford-West TBH, Coward SRL, Notarberardino B, Walker B, Abdul-Aziz A. 2008. An efficient approach to converting three-dimensional image data into highly accurate computational models. Philos Trans R Soc A Math Phys Eng Sci. 366:315573. 
Zhang L, Yang KH and King AI. A proposed injury threshold for mild traumatic brain injury. Journal of Biomechanical Engineering 2004; 126(2): 226-236.

\section{Appendix A \\ Material properties and shell thicknesses used in NRL-Simpleware head model.}

Table A1. Head model components, constitutive models and calibrated material parameters.

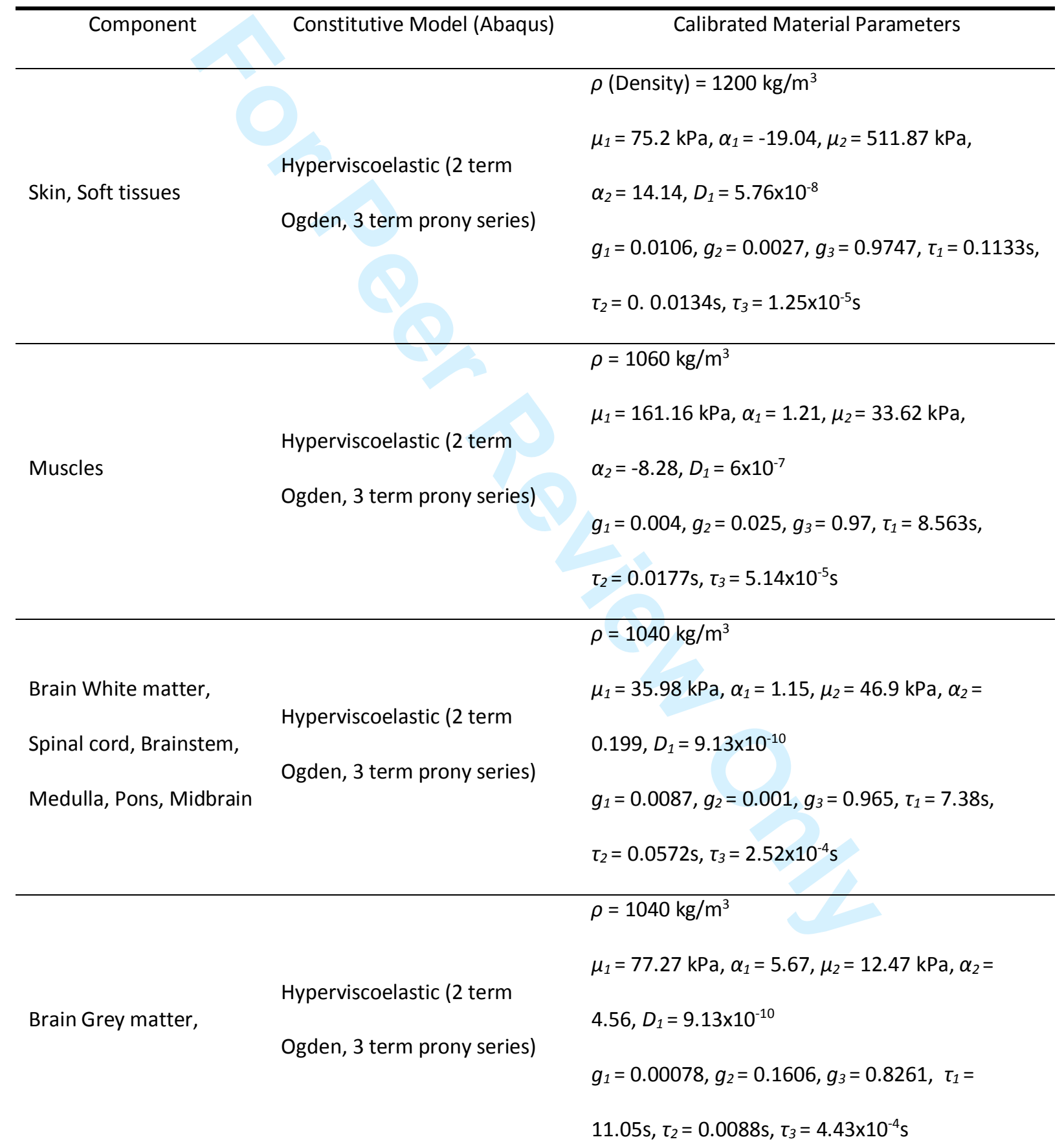


Cerbral Spinal Fluid,

$\begin{array}{lll}\text { Eyes, Ventricles, Veins } & \text { Hyperelastic (Neo-Hookean) } & \rho=1040 \mathrm{~kg} / \mathrm{m}^{3} \\ \text { (interior) } & C_{10}=11.27 \mathrm{kPa}, D_{1}=9.13 \times 10^{-10}\end{array}$

Falx cerebri, Tentorium

cerebelli,

Hyperelastic (Ogden)

$\rho=1130 \mathrm{~kg} / \mathrm{m}^{3}$

Dura Mater

$\mu_{1}=1.75 \mathrm{MPa}, \alpha_{1}=21.28, D_{1}=2.28 \times 10^{-8}$

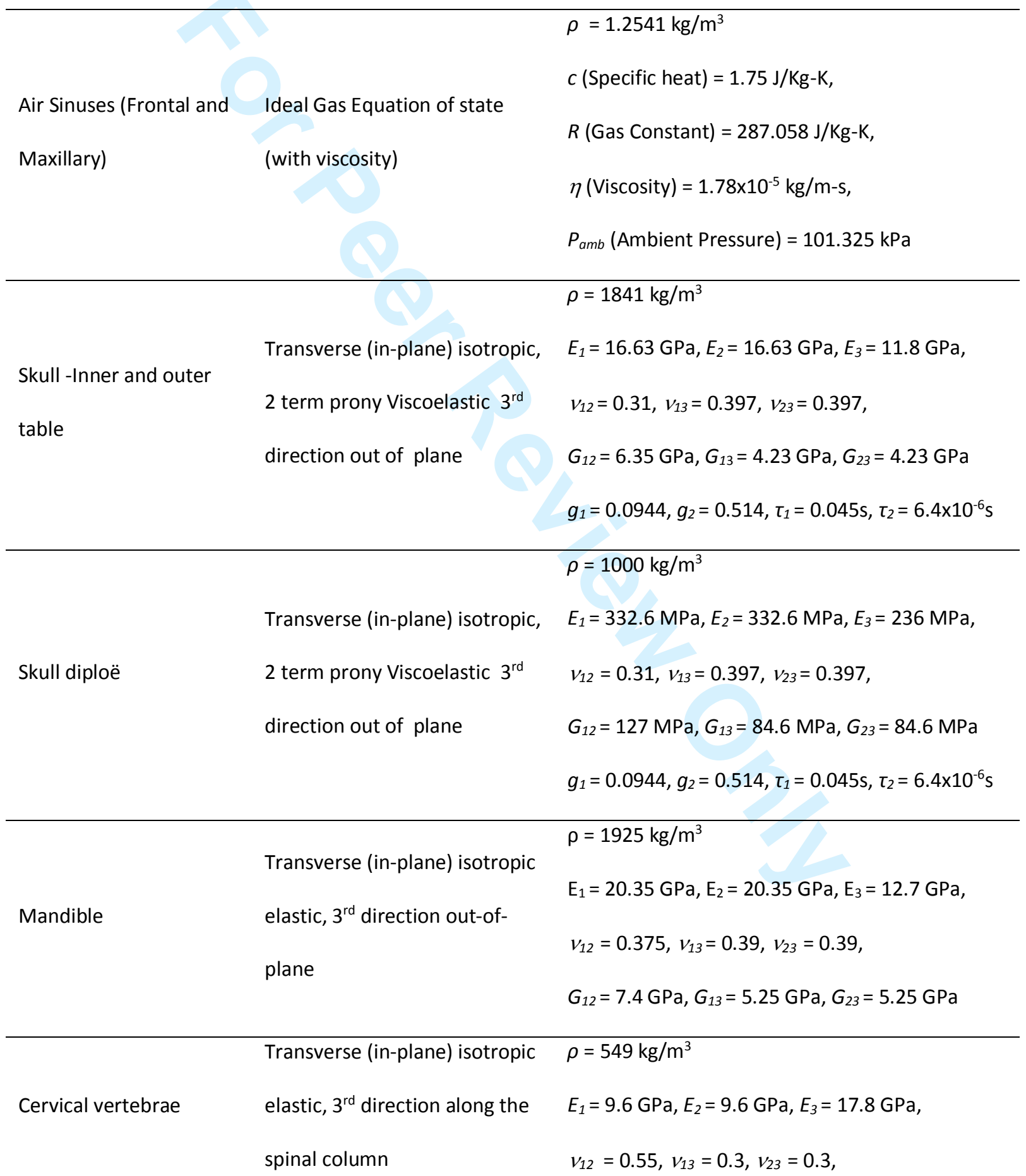




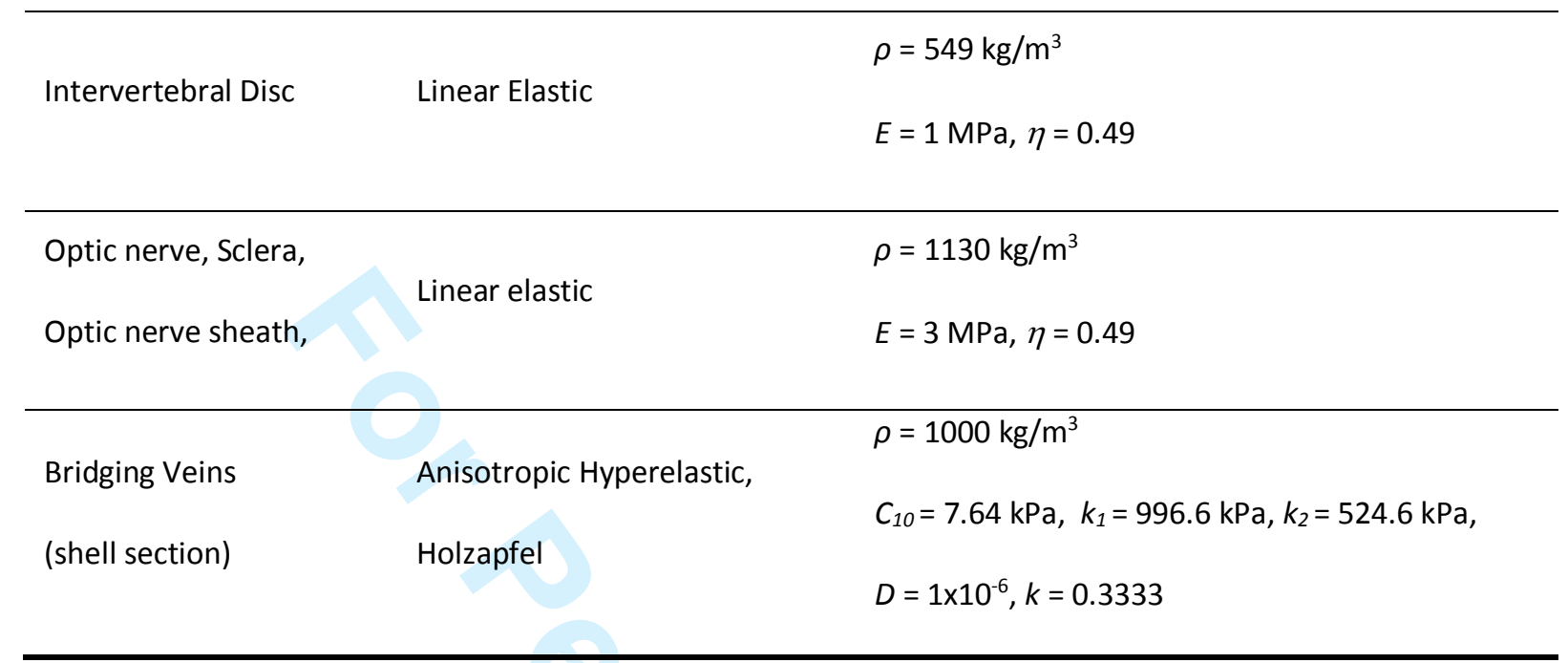

Table A2. Assigned thicknesses for head membranes (shell elements).

\begin{tabular}{ll}
\hline \multicolumn{1}{c}{ Component } & Thickness $(\mathrm{mm})$ \\
\hline Dura mater & 0.55 \\
Sclera & 0.67 \\
Optic nerve sheath & 0.06 \\
Bridging Veins & 0.12 \\
\hline
\end{tabular}




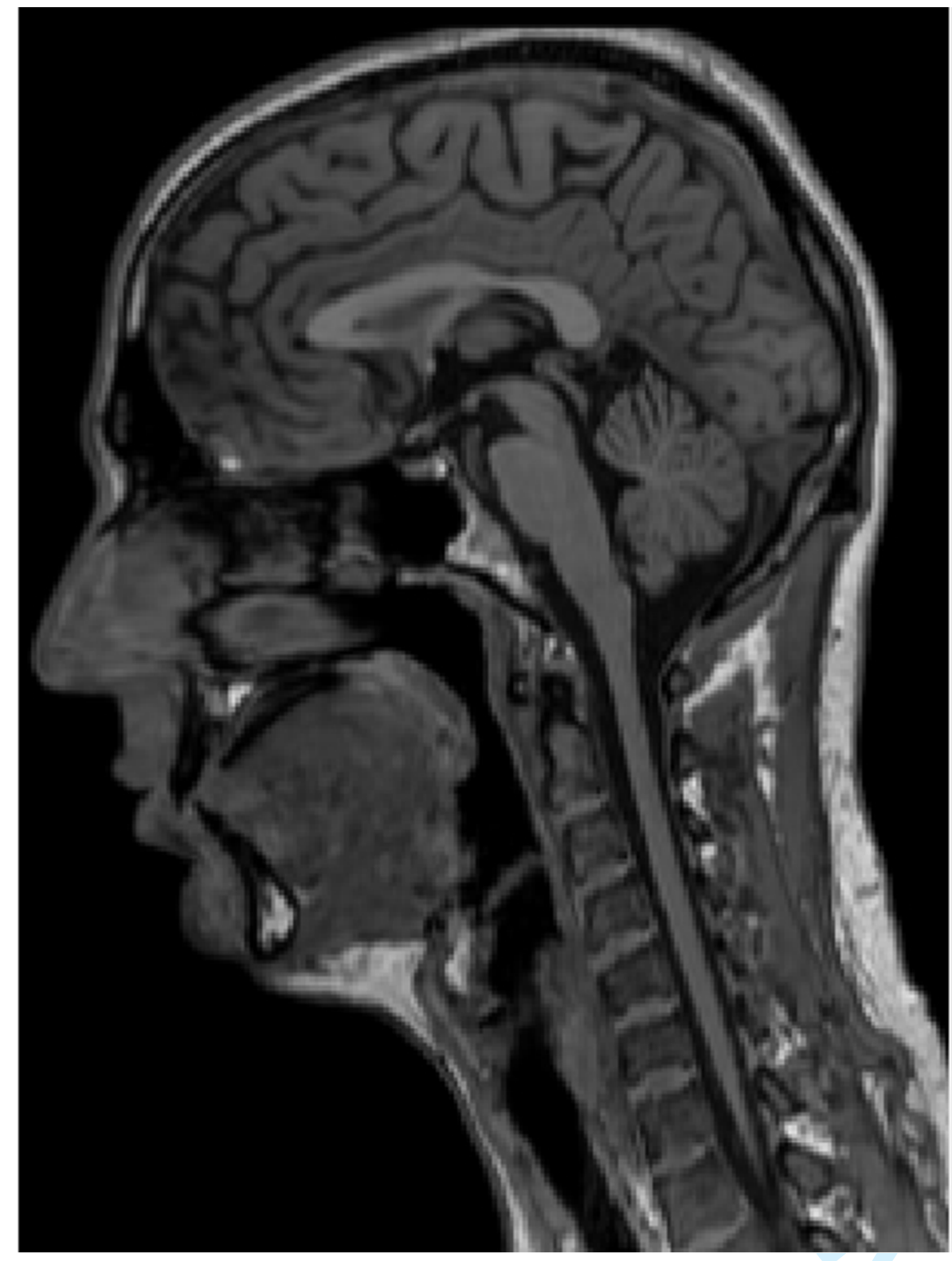




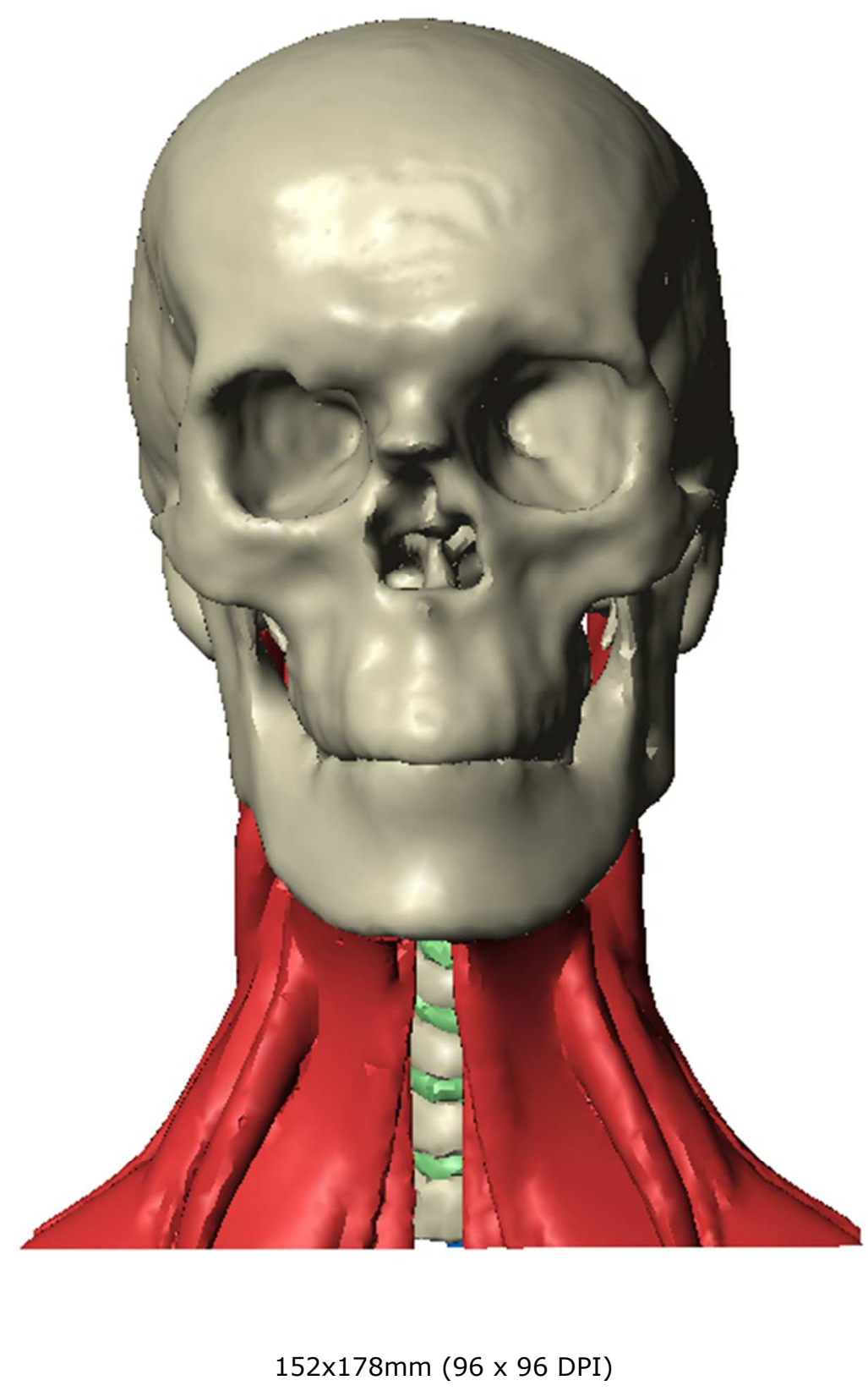




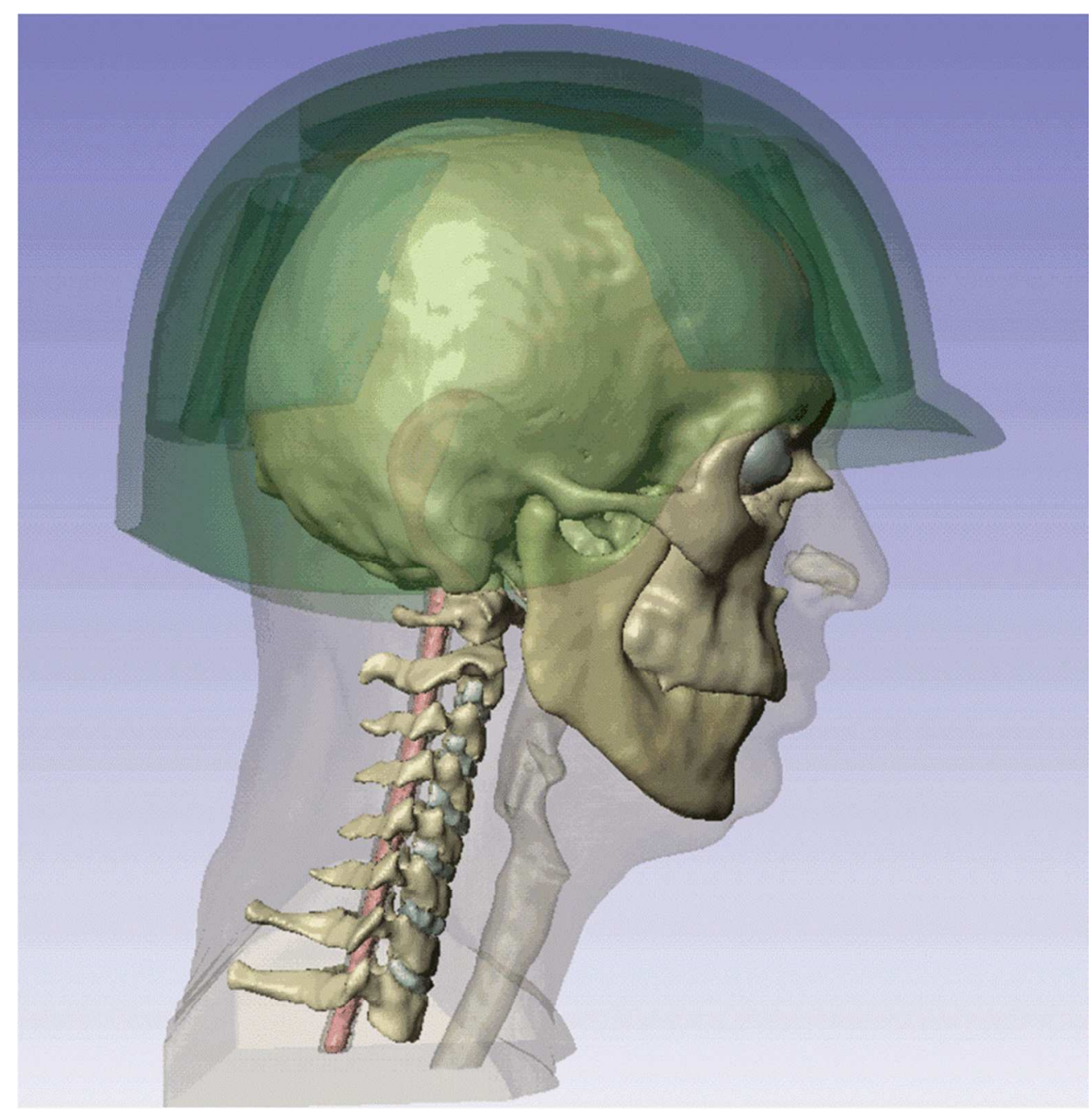

$279 \times 286 \mathrm{~mm}(96 \times 96 \mathrm{DPI})$ 


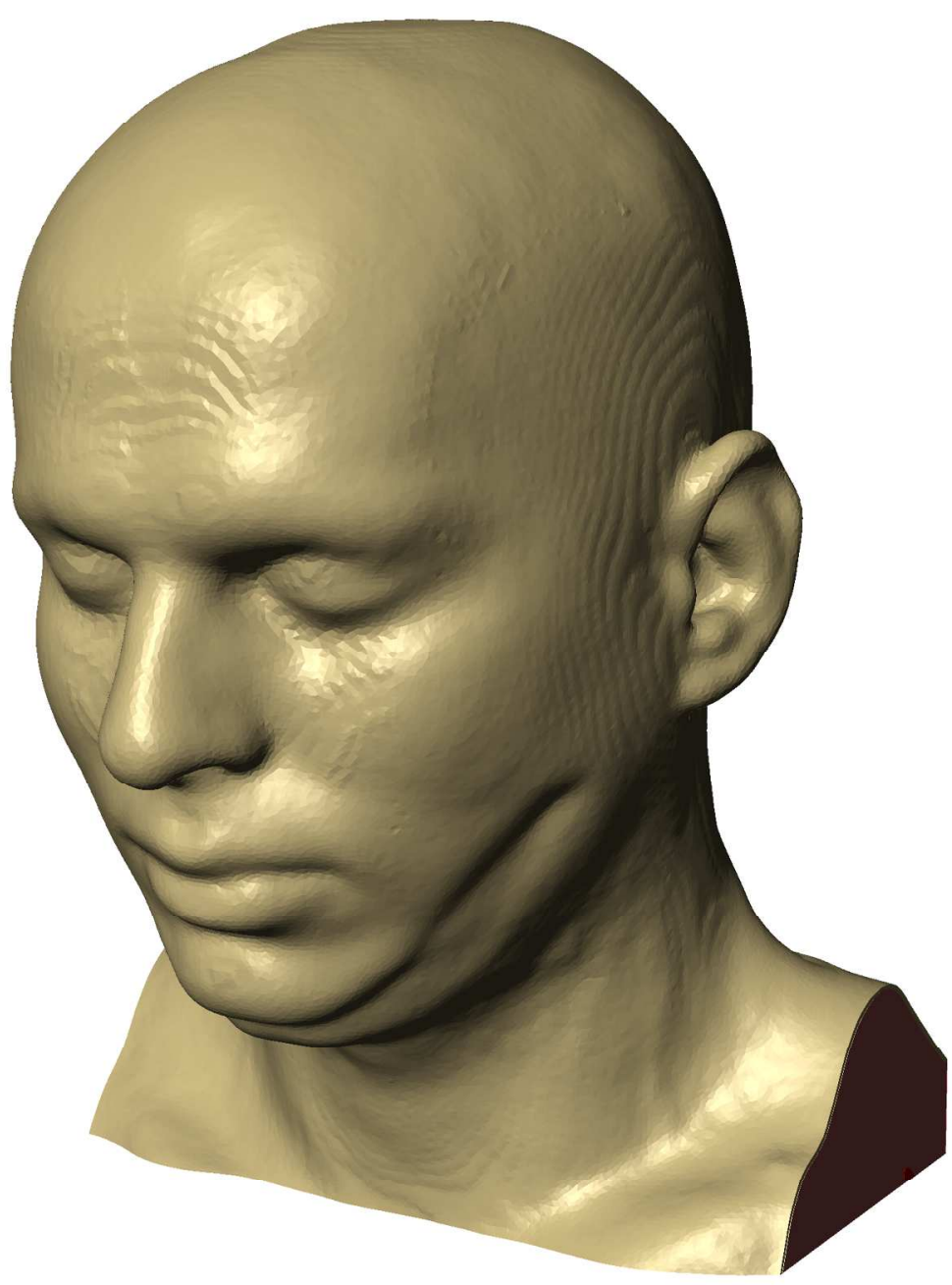

674x695mm (72 x 72 DPI $)$ 


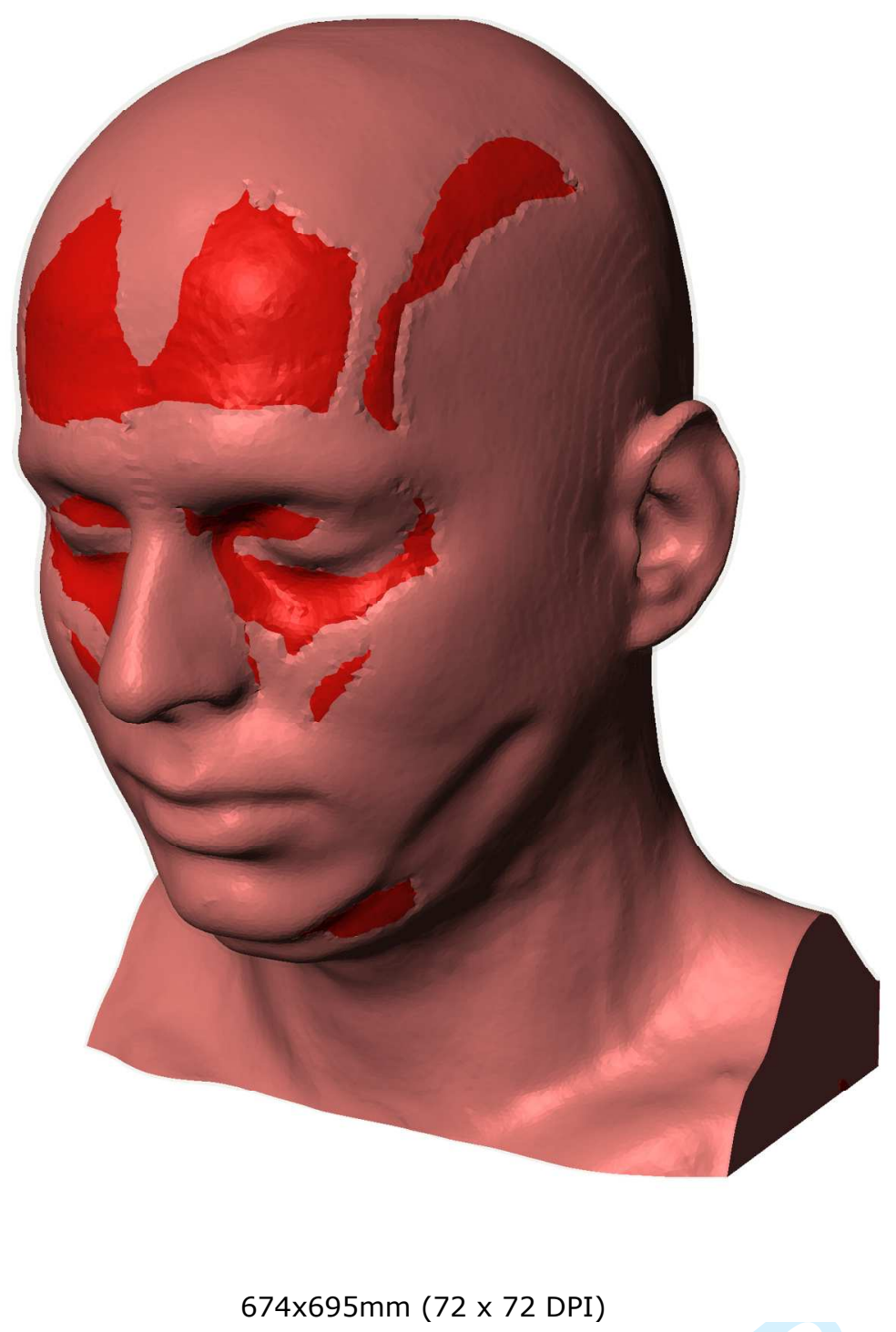




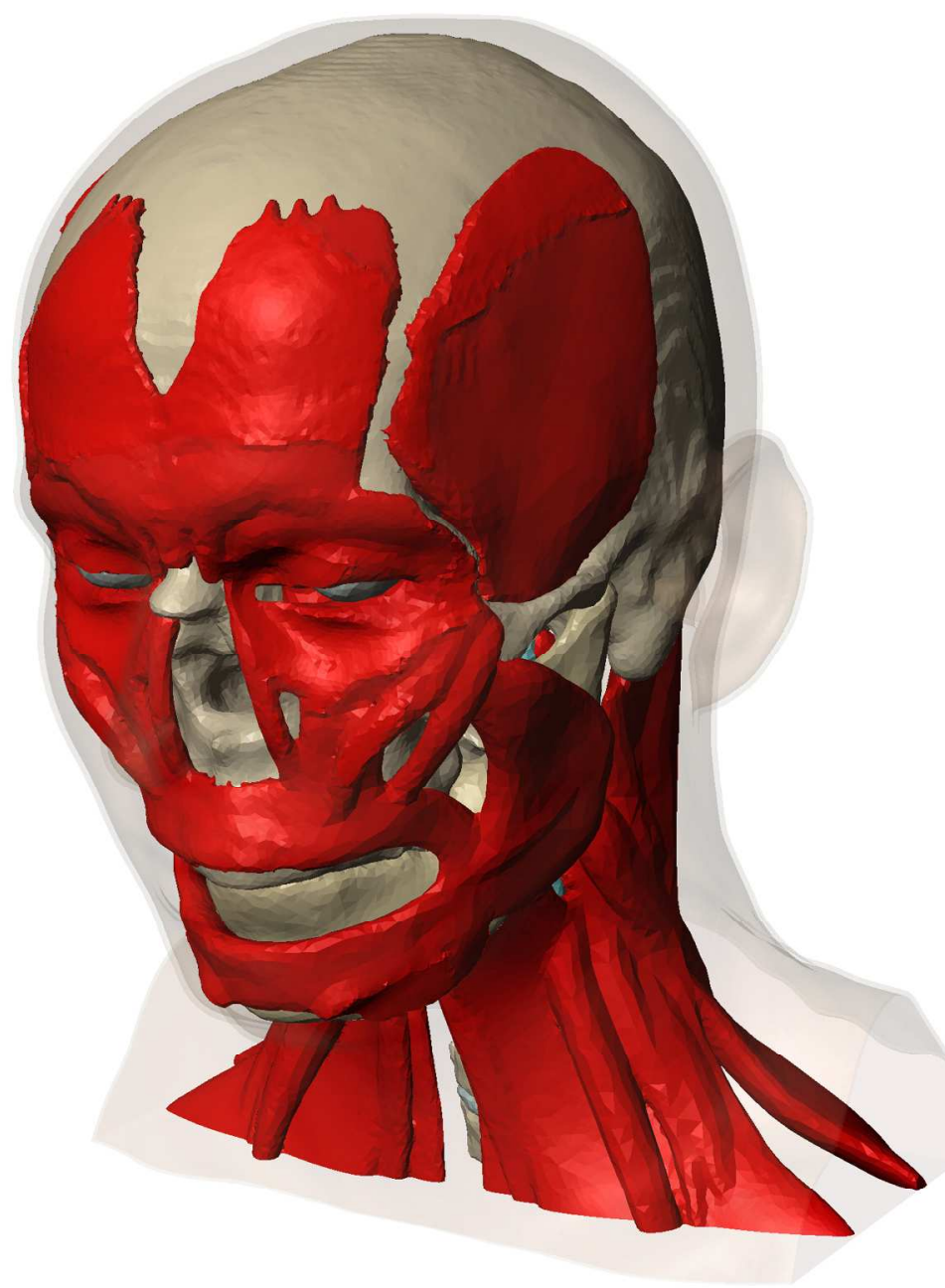

$674 \times 695 \mathrm{~mm}(72 \times 72$ DPI $)$ 


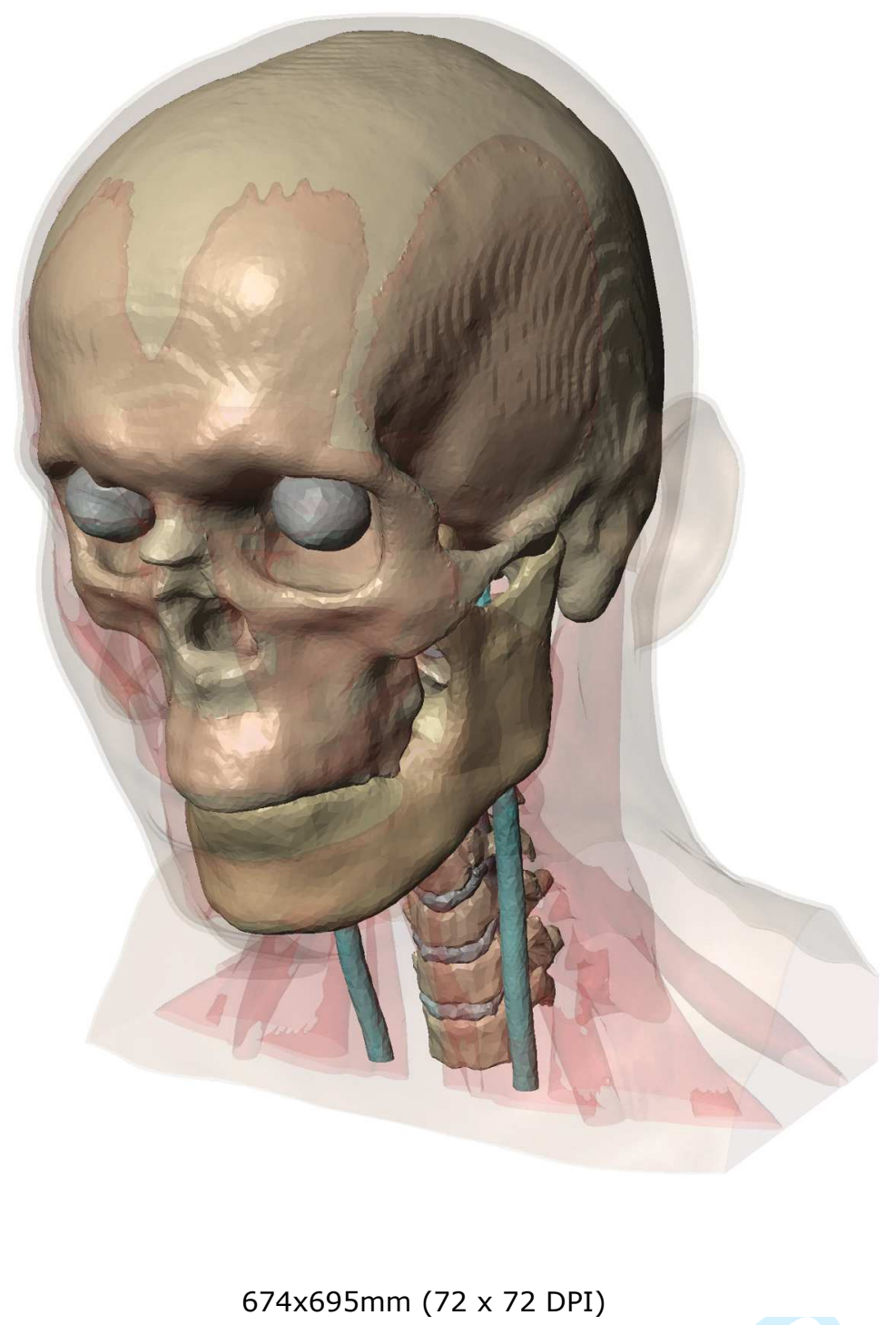

(7) 


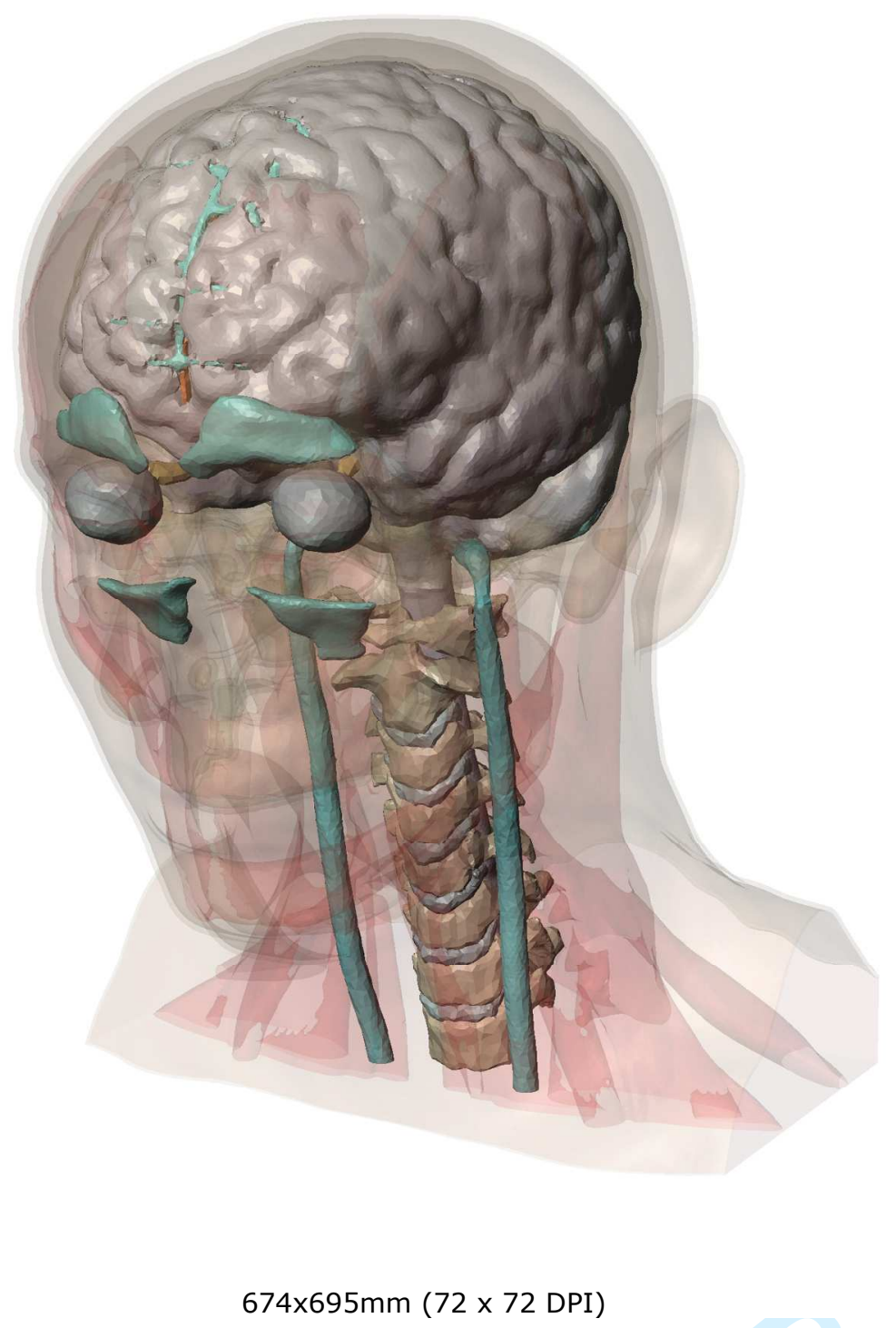

URL: http://mc.manuscriptcentral.com/gcmb 


1
2
3
4
5
6
7
8
9
10
11
12
13
14
15
16
17
18
19
20
21
22
23
24
25
26
27
28
29
30
31
32
33
34
35
36
37
38
39
40
41
42
43
44
45
46
47
50
58
59
50
52
53
50

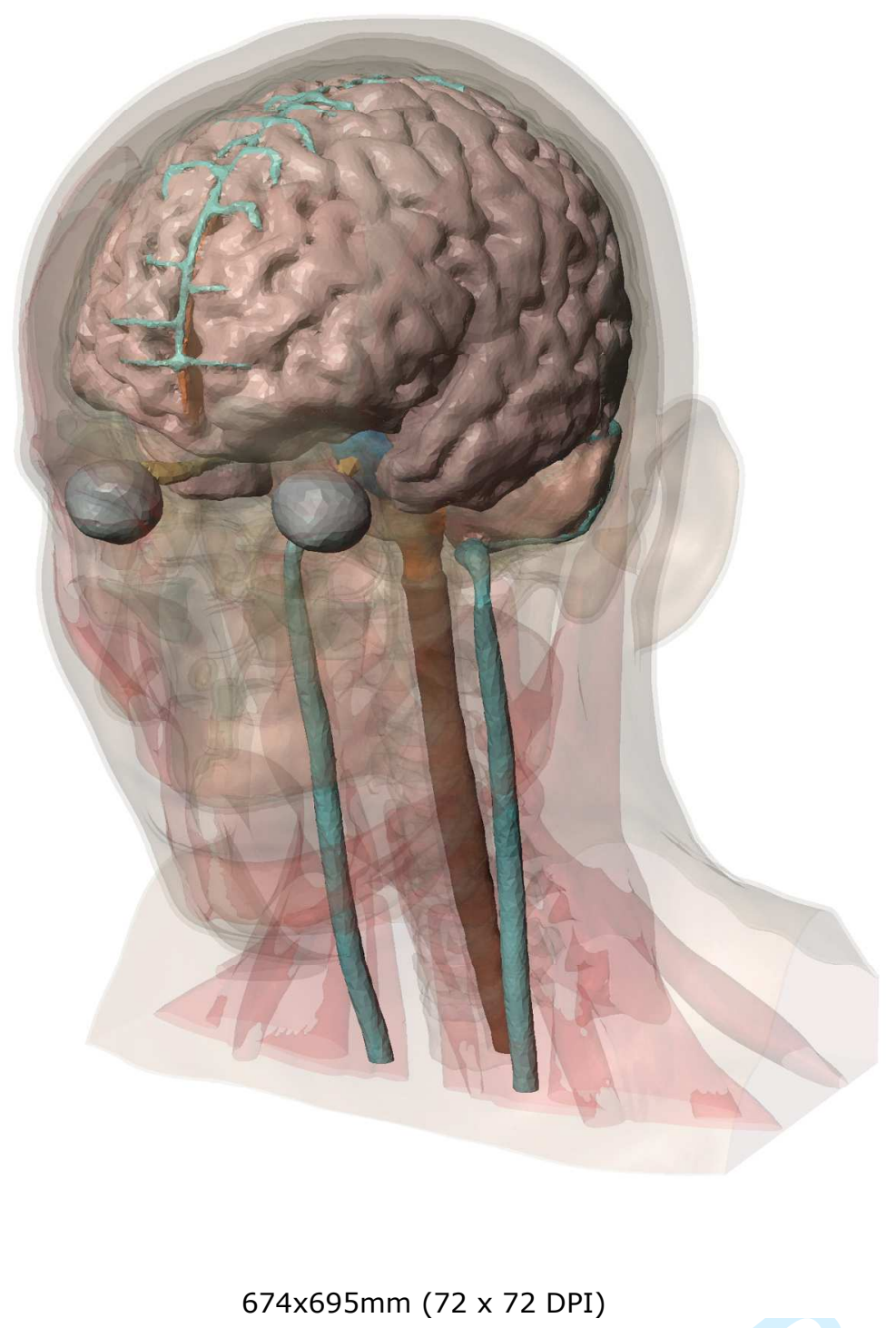

(72 $\times 72$ DPI $)$ 


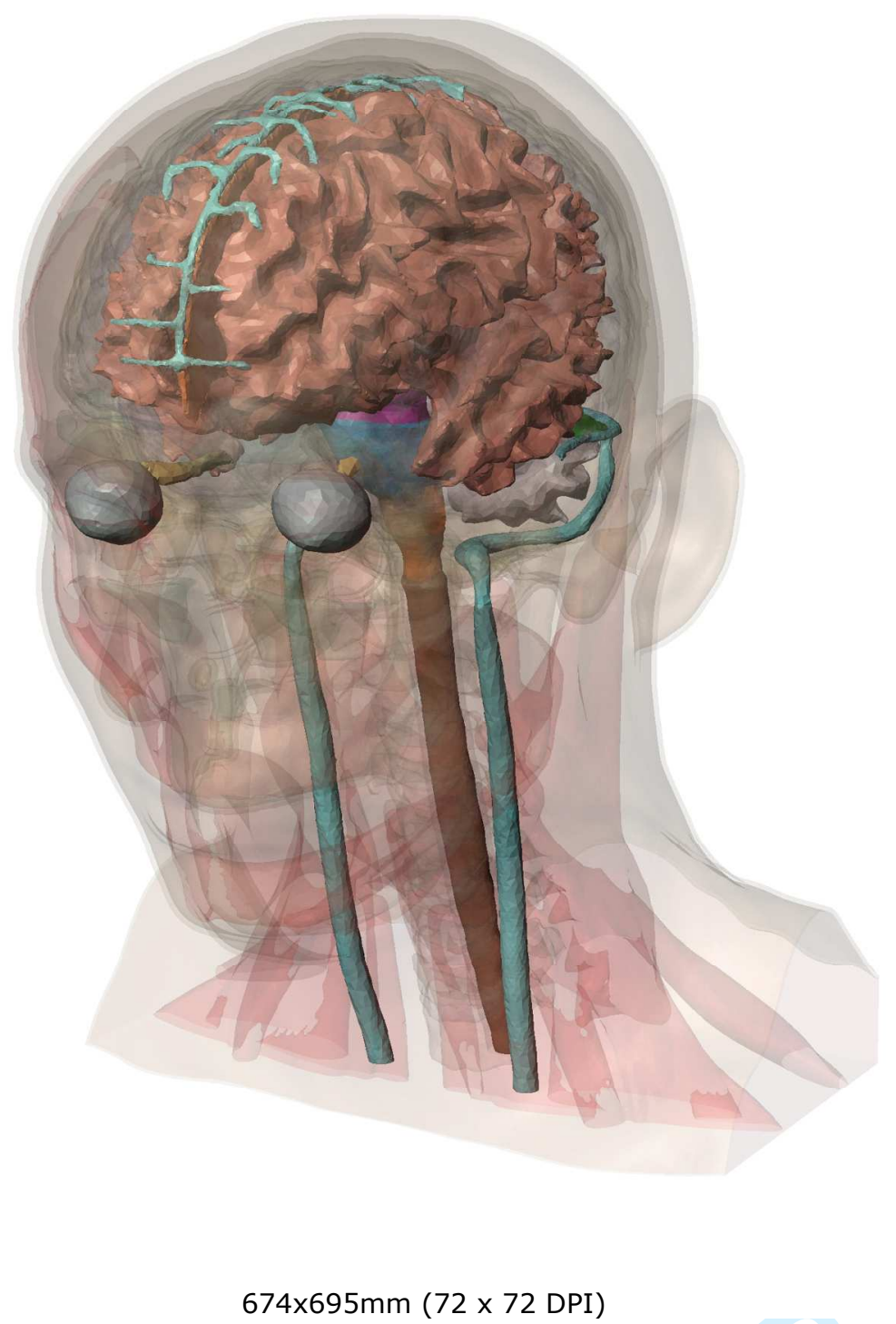




1
1
3
4
5
6
7
8
9
10
11
12
13
14
15
16
17
18
19
20
21
22
23
24
25
26
27
28
29
30
31
32
33
34
35
36
37
38
39
40
41
42
43
44
55
50
56
57
48
59
50
51
53
50

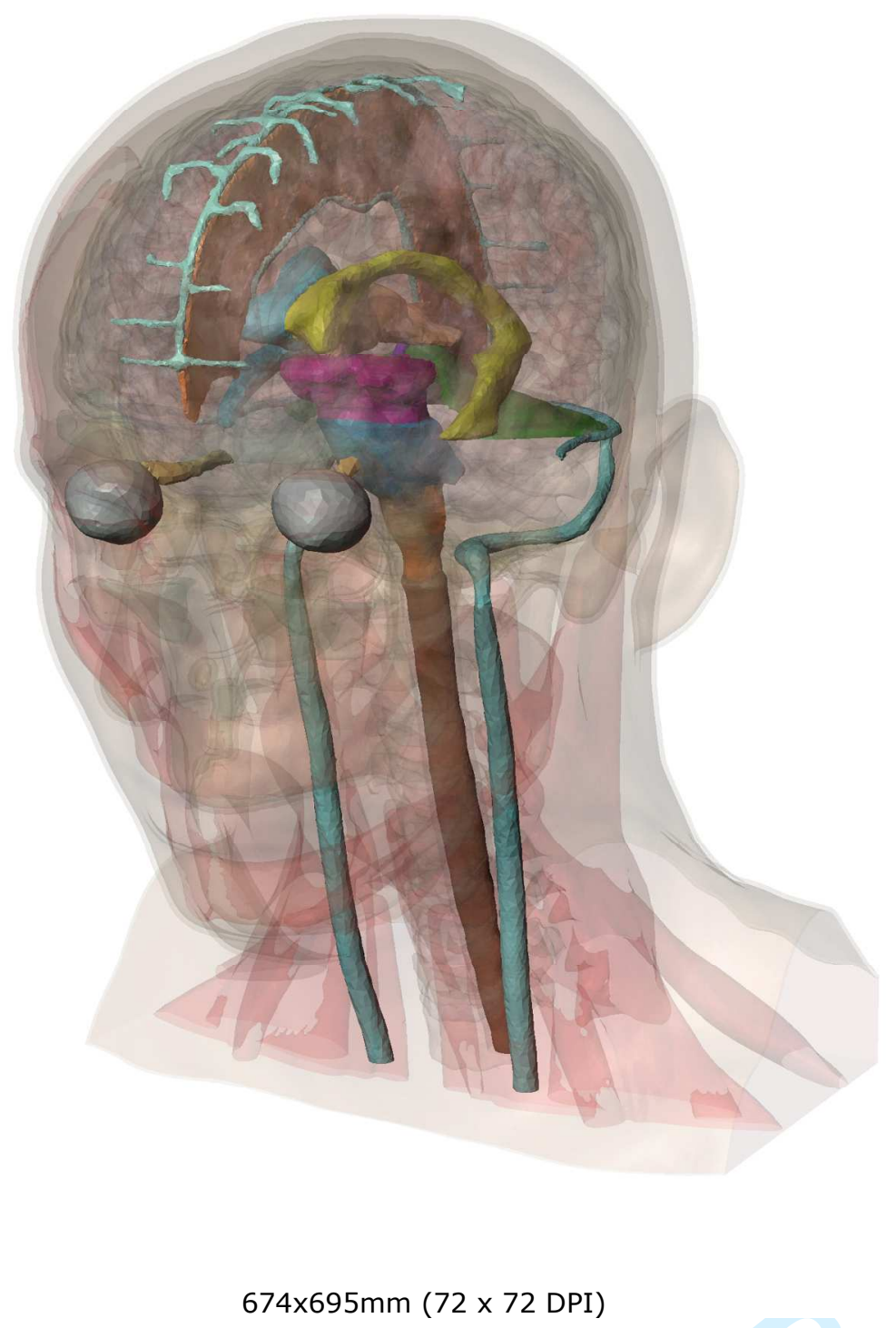

URL: http://mc.manuscriptcentral.com/gcmb 


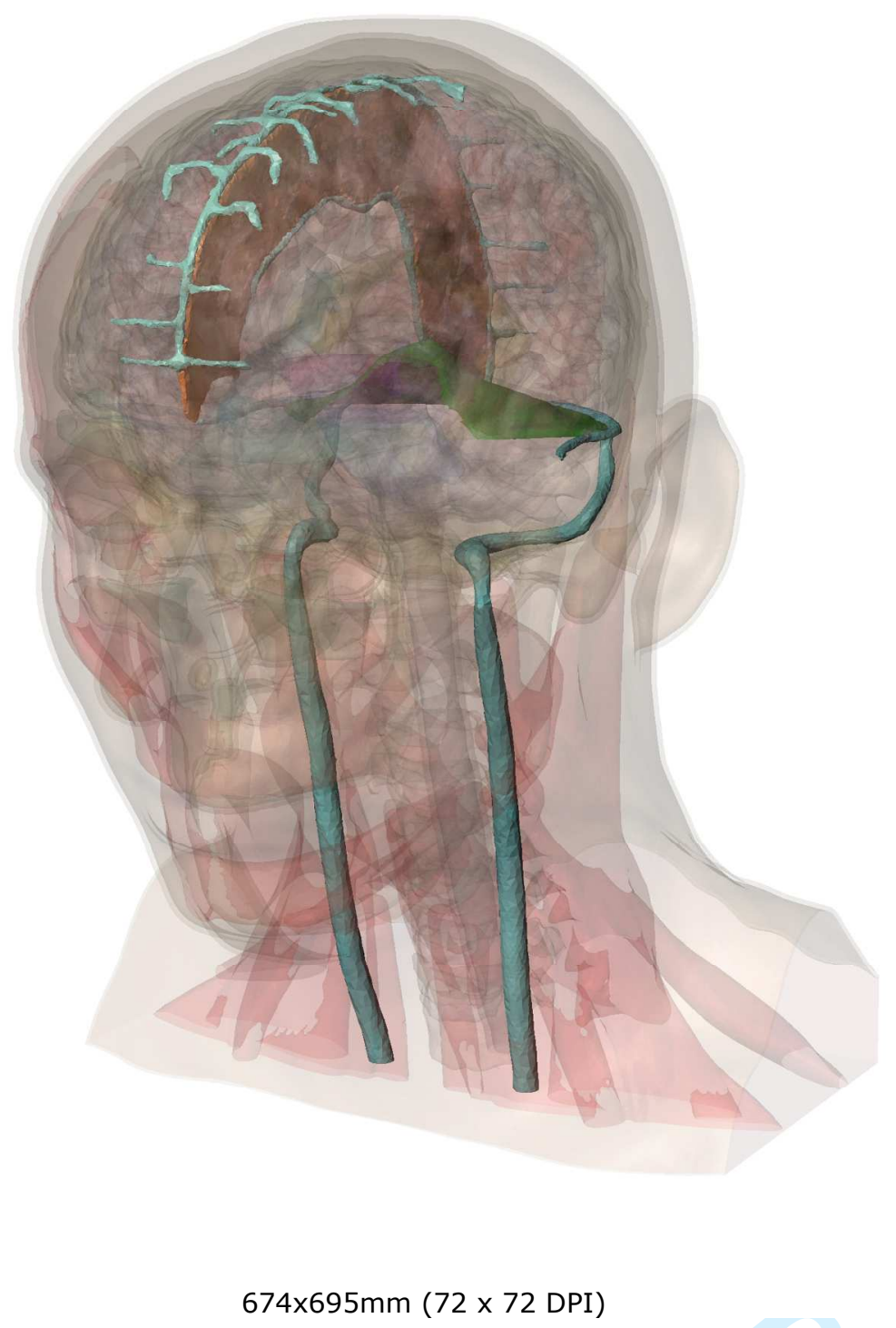




1
2
3
4
5
6
7
8
9
10
11
12
13
14
15
16
17
18
19
20
21
22
23
24
25
26
27
28
29
30
31
32
33
34
35
36
37
38
39
40
41
42
43
44
45
46
47
50
58
59
50
52
53
50

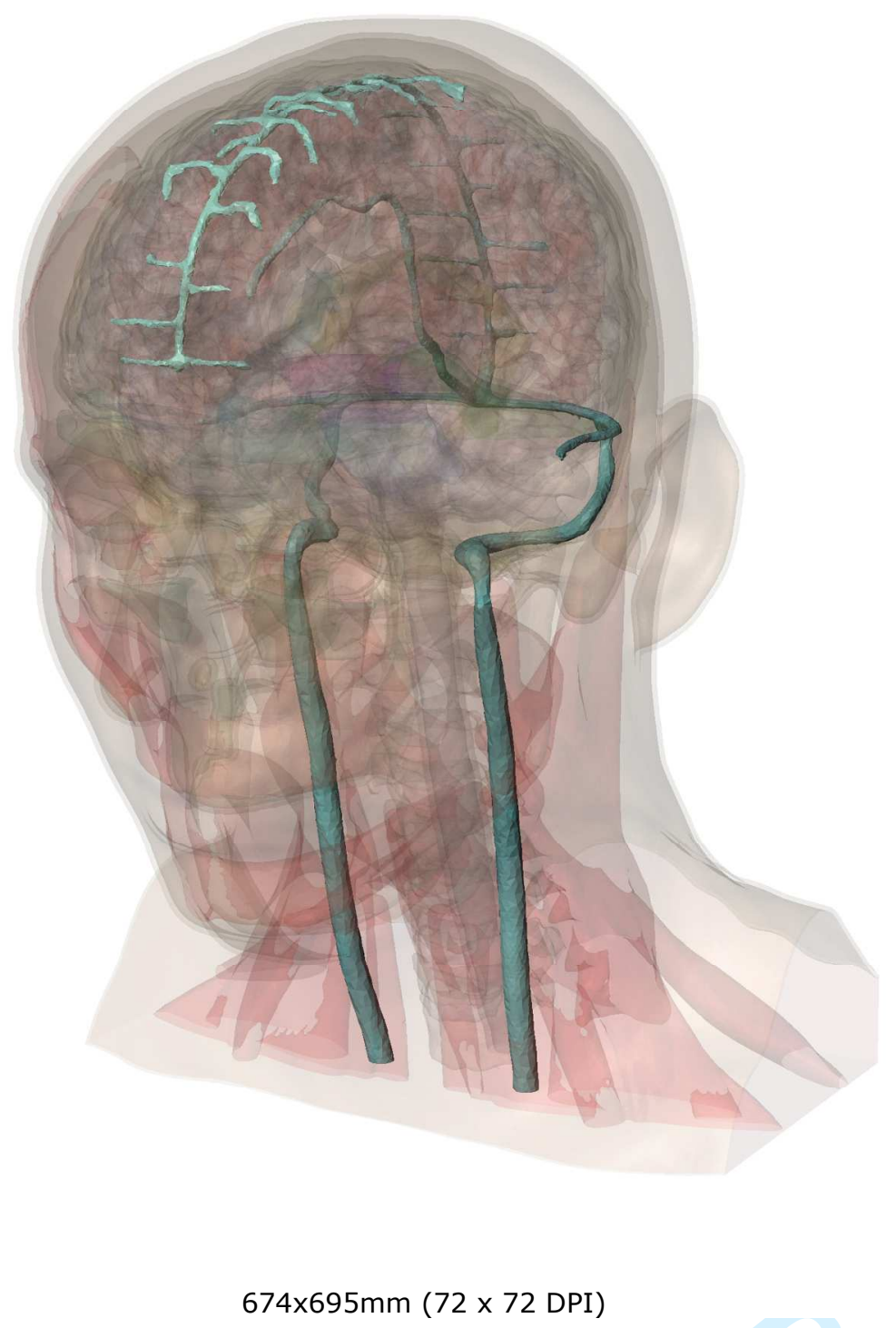

URL: http://mc.manuscriptcentral.com/gcmb 


1
1
3
4
5
6
7
8
9
10
11
12
13
14
15
16
17
18
19
20
21
22
23
24
25
26
27
28
29
30
31
32
33
34
35
36
37
38
39
40
41
42
43
44
55
50
56
57
48
59
50
51
53
50

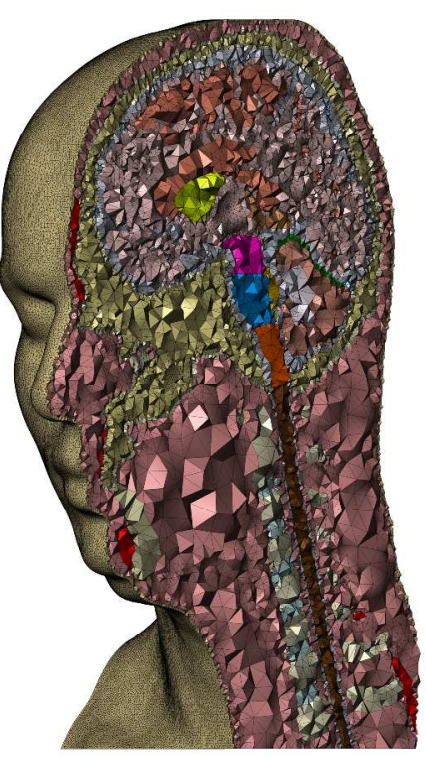

$2242 \times 1289 \mathrm{~mm}(72 \times 72$ DPI $)$

29

31

32

33

34

35

36

37

39

40

41

42

44

45

46

47

48

49

51

52

53

54

55

57

58

59

60

URL: http://mc.manuscriptcentral.com/gcmb 


1
2
3
4
5
6
7
8
9
10
11
12
13
14
15
16
17
18
19
20
21
22
23
24
25
26
27
28
29
30
31
32
33
34
35
36
37
38
39
40
41
42
43
44
45
46
47
48
49
50
51
52
53
54
55
56
57
58
59
60

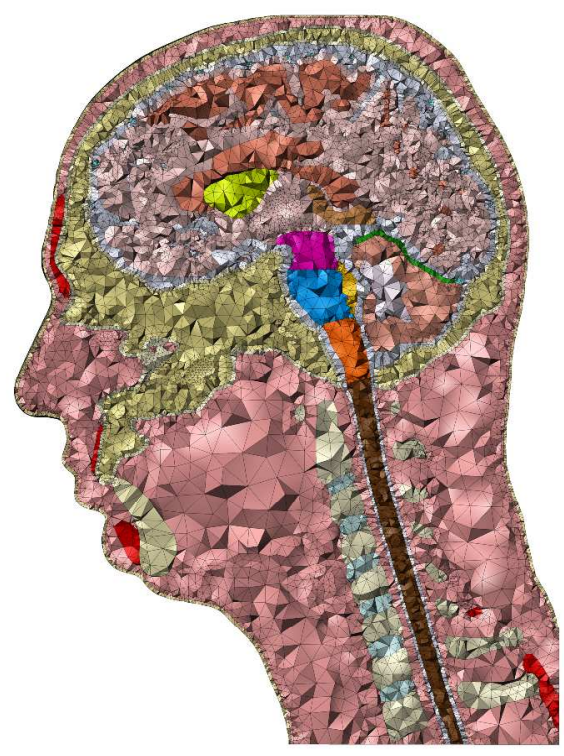

$2242 \times 1289 \mathrm{~mm}(72 \times 72 \mathrm{DPI})$

29

31

32

33

34

35

36

39

40

41

42

43
44

45

46

47

48

49

50

52

53

54

55

56

57

58

59
60

URL: http://mc.manuscriptcentral.com/gcmb 


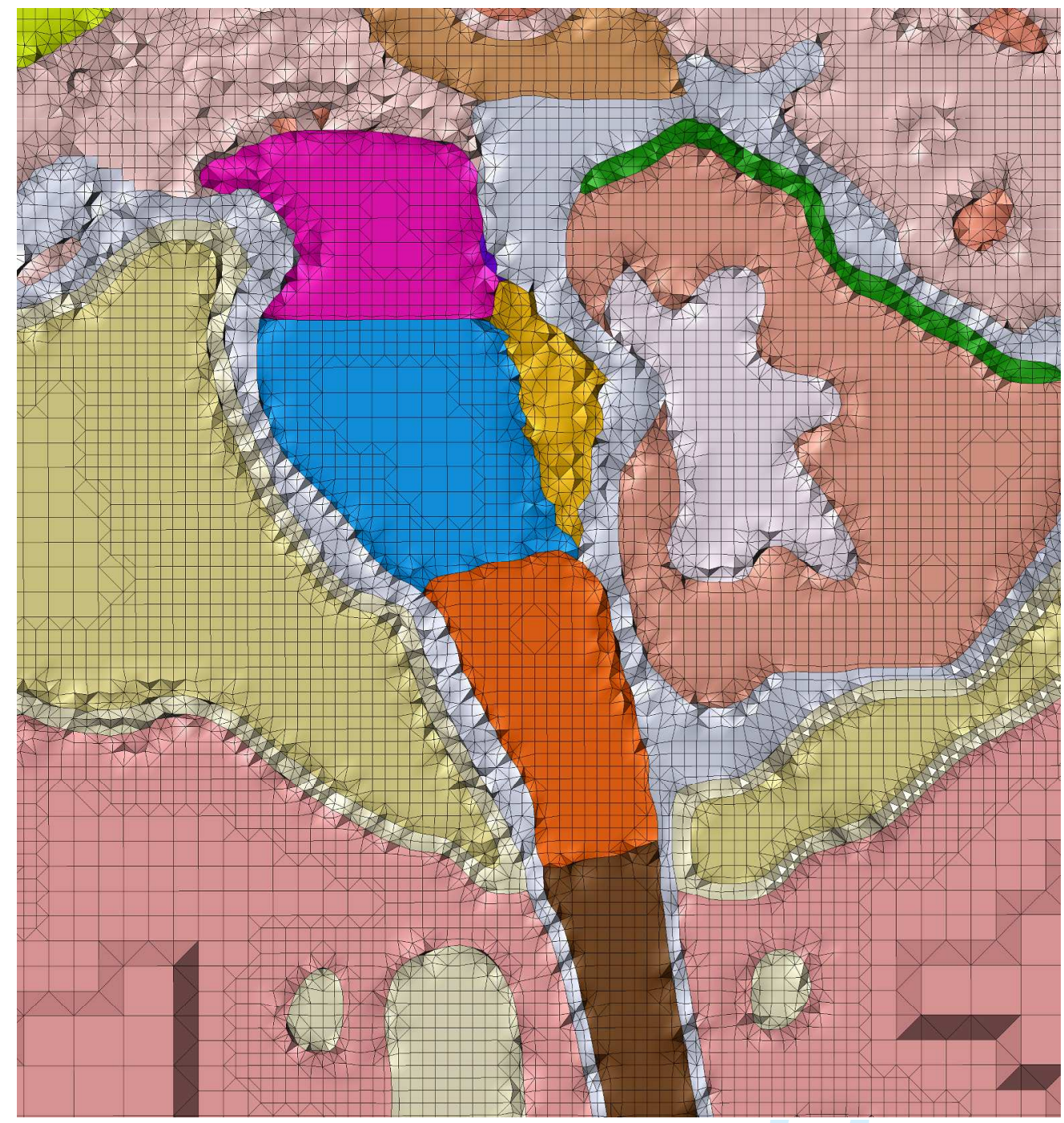




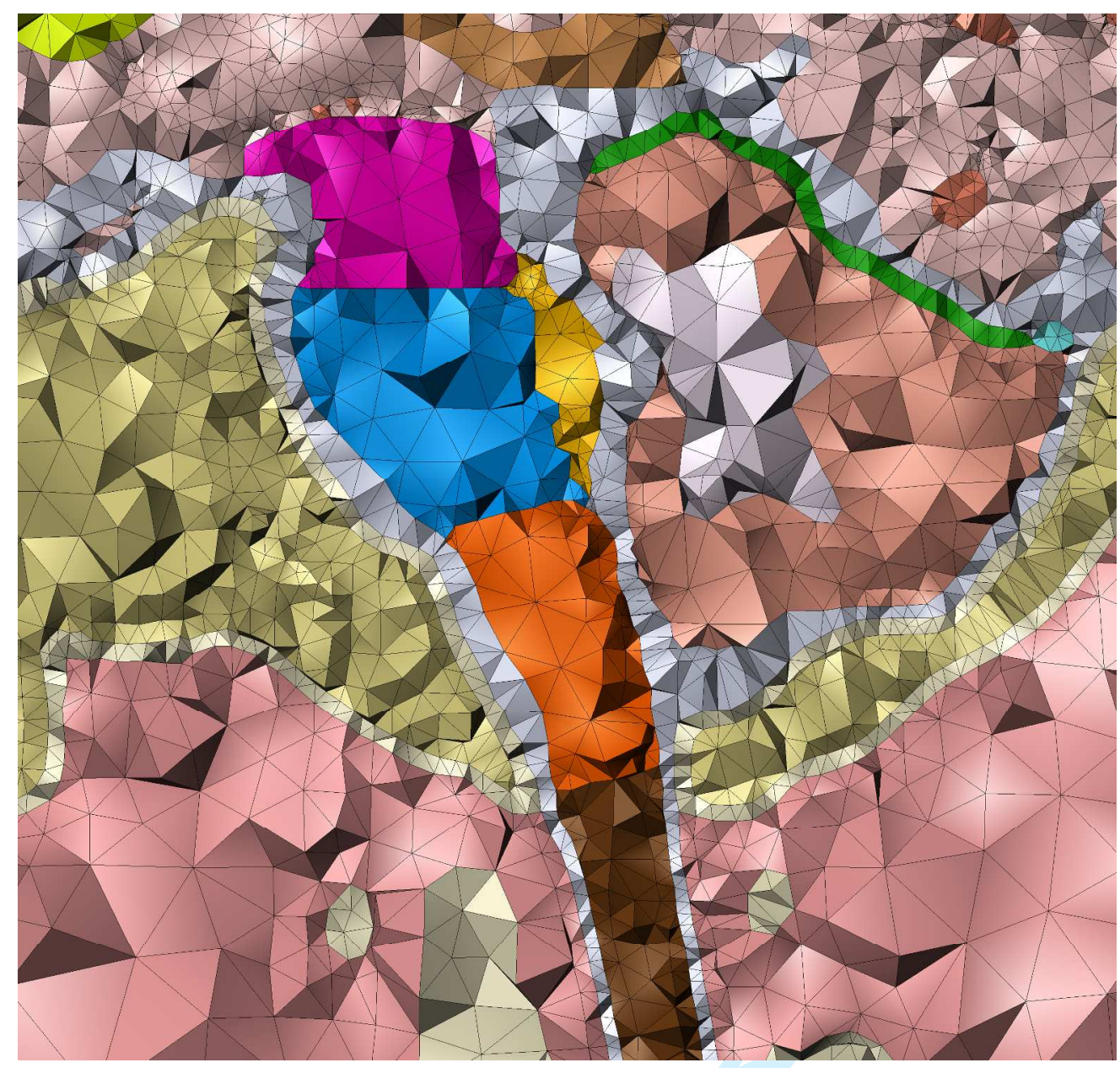



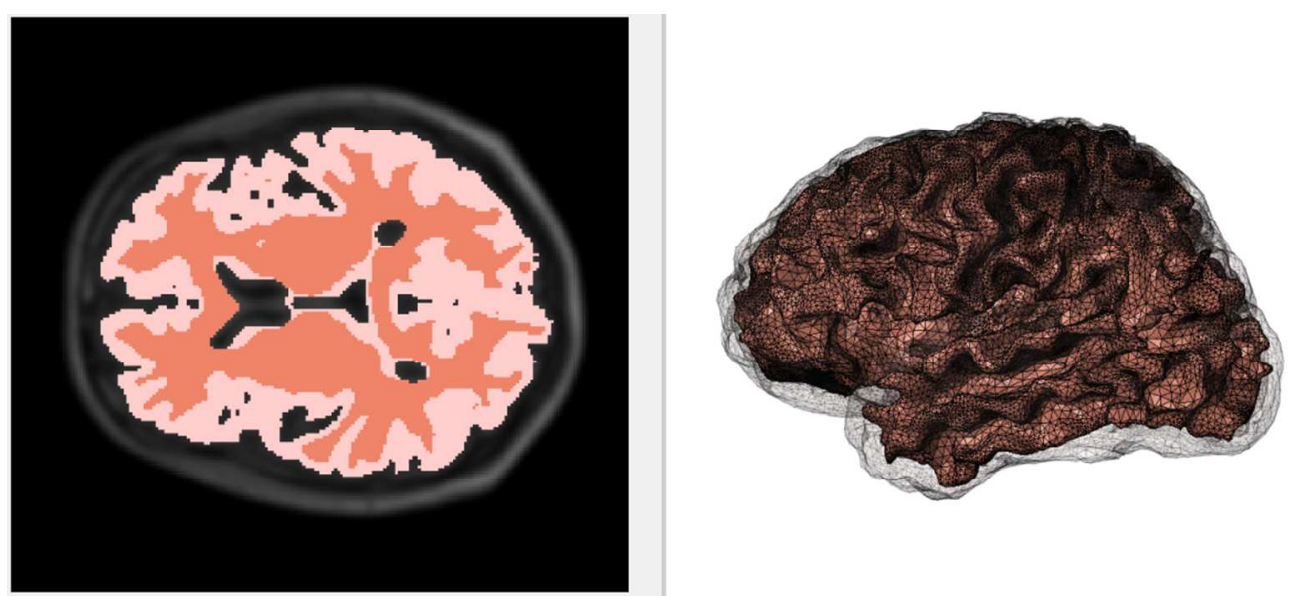

$136 \times 60 \mathrm{~mm}(220 \times 220$ DPI $)$

1

2

3

4

5

6
7

8

9

10

11

12

13

15

16

17

18

19

20

.

24

25

26

27

28

29

30

31

32

33

34

35

36

37

38

39

40

41

42

43

44

45

46

47

48

49

50

51

52

53

54

55

56

57

58

59

60 

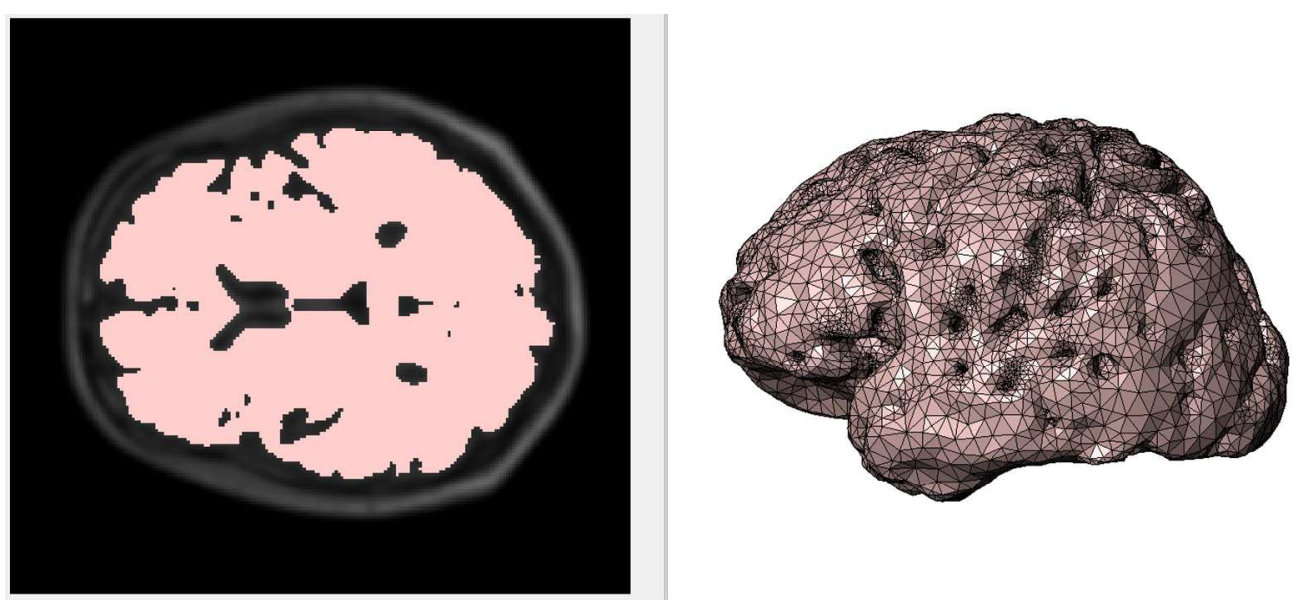

$398 \times 179 m m(96 \times 96$ DPI) 


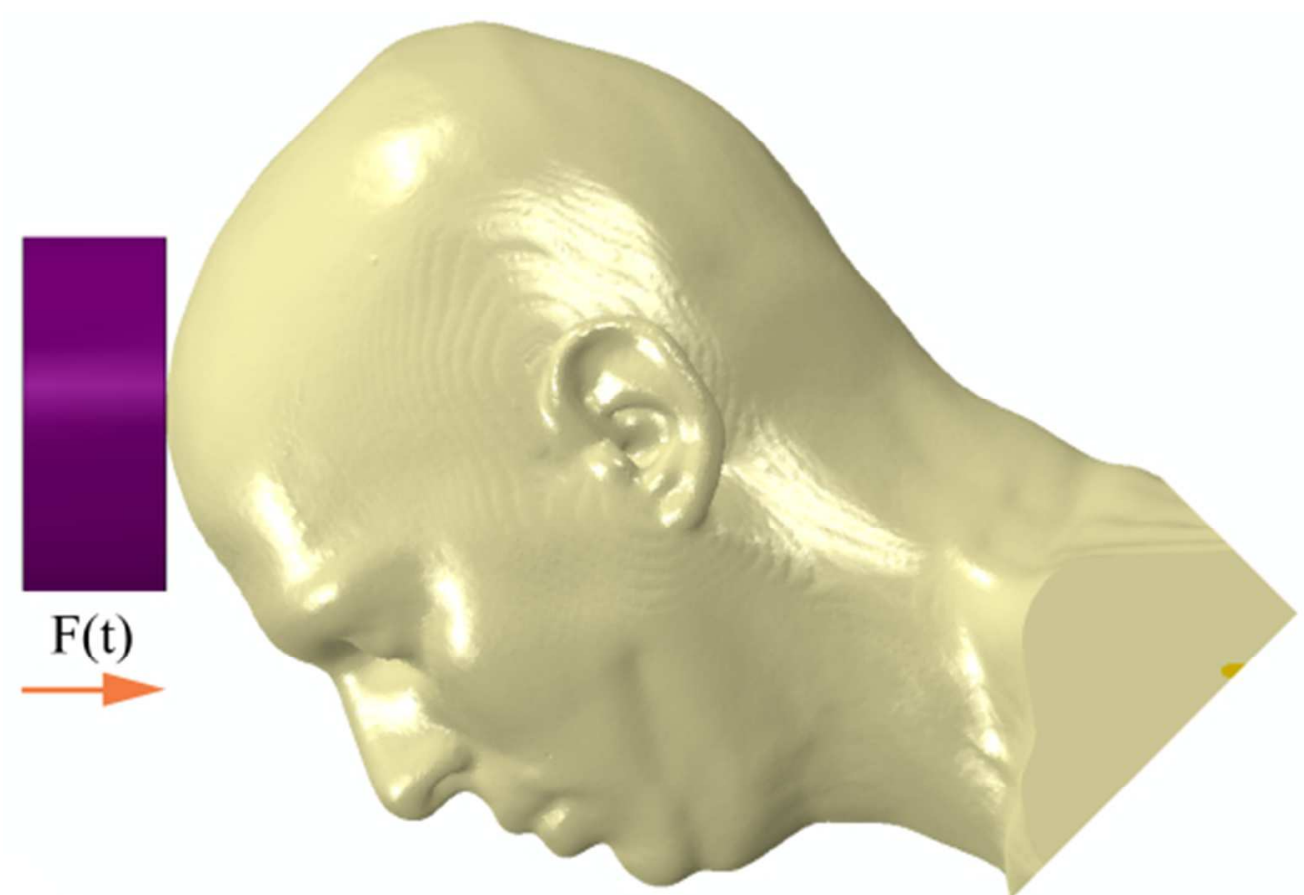

$62 \times 43 \mathrm{~mm}(220 \times 220 \mathrm{DPI})$ 


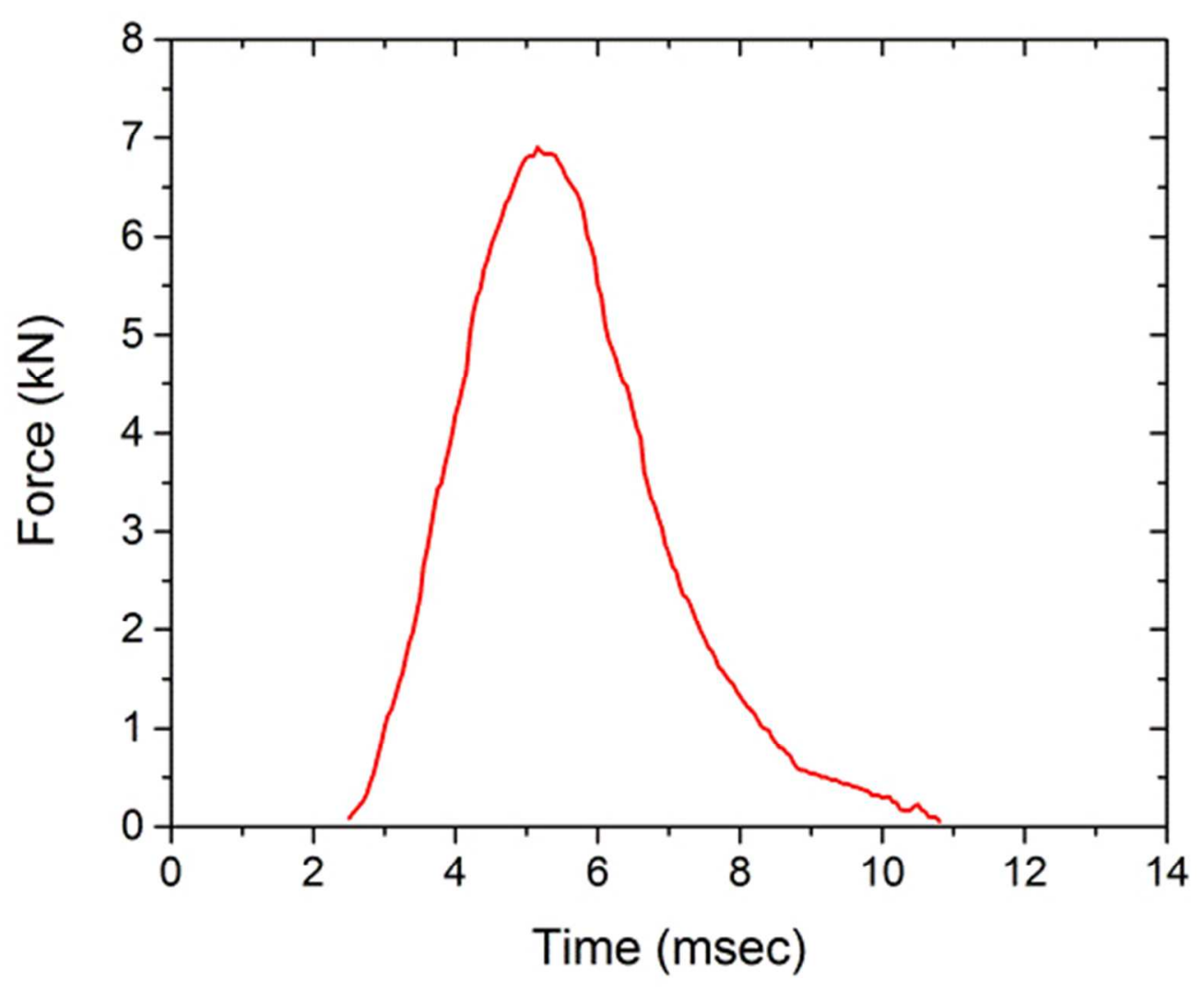

$116 \times 94 \mathrm{~mm}(120 \times 120$ DPI $)$ 


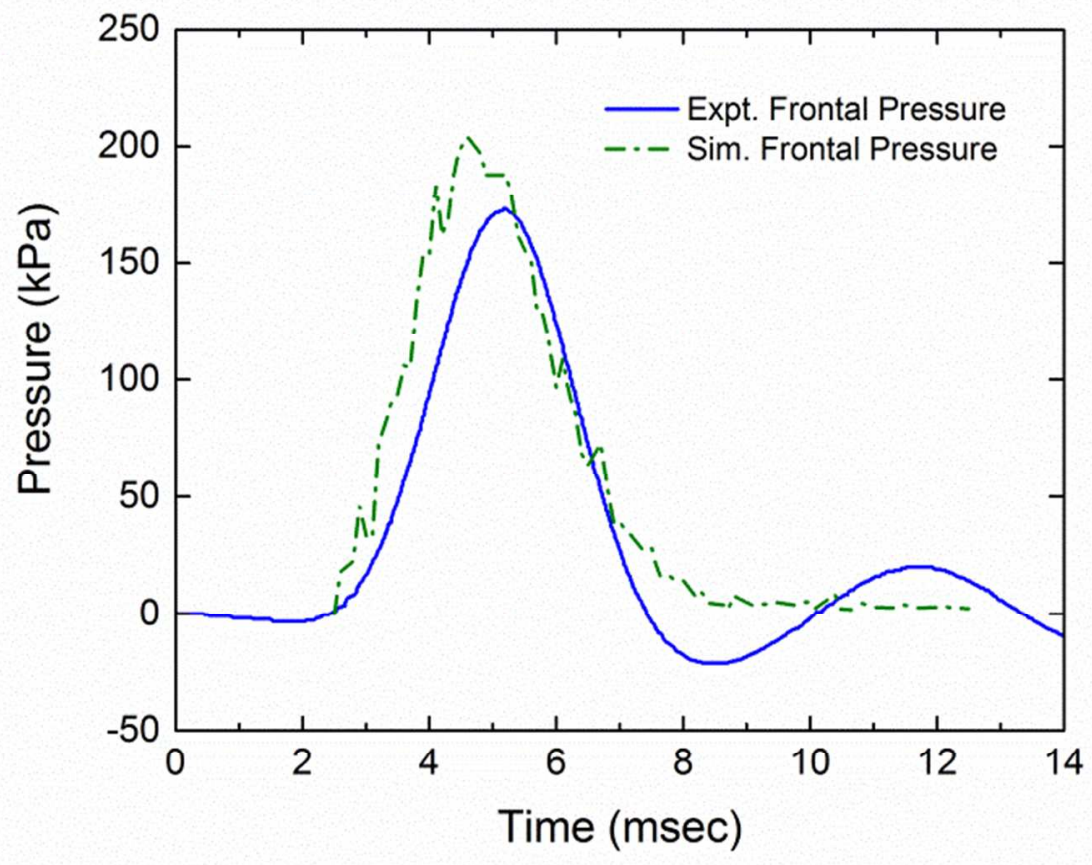

$169 \times 127 \mathrm{~mm}(120 \times 120 \mathrm{DPI})$ 


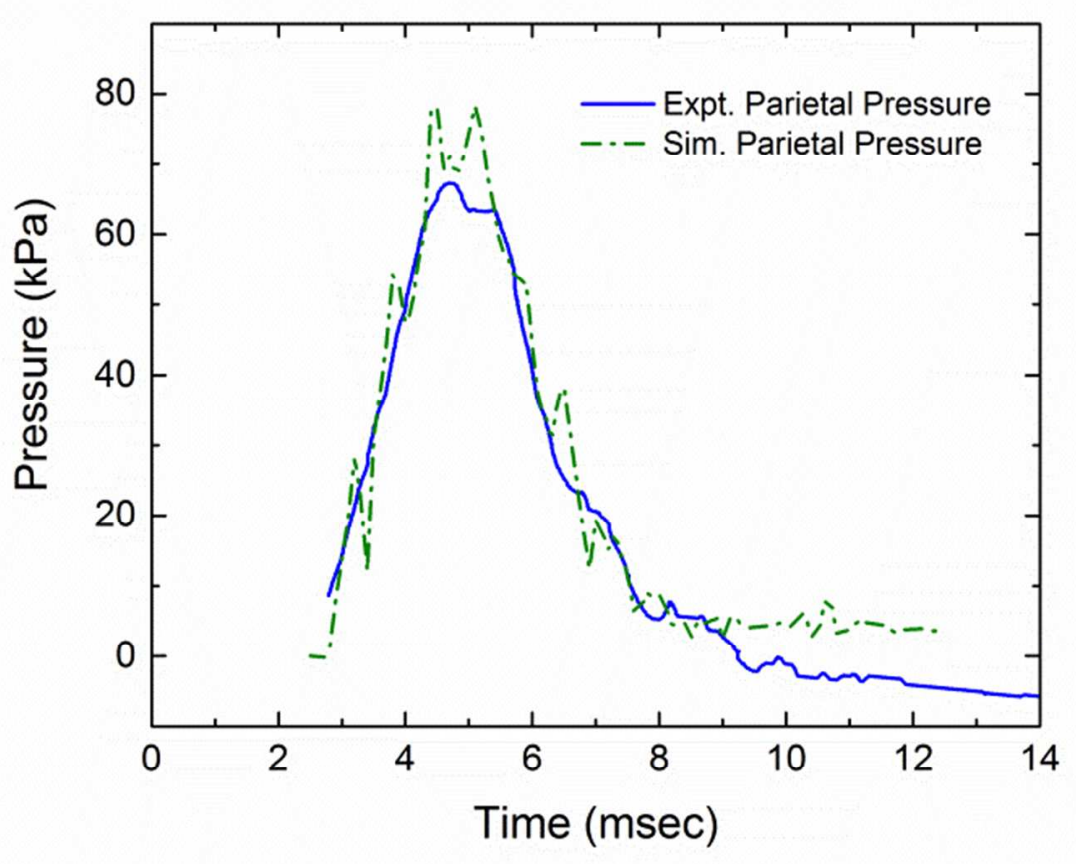

$169 \times 127 \mathrm{~mm}(120 \times 120 \mathrm{DPI})$

URL: http://mc.manuscriptcentral.com/gcmb 


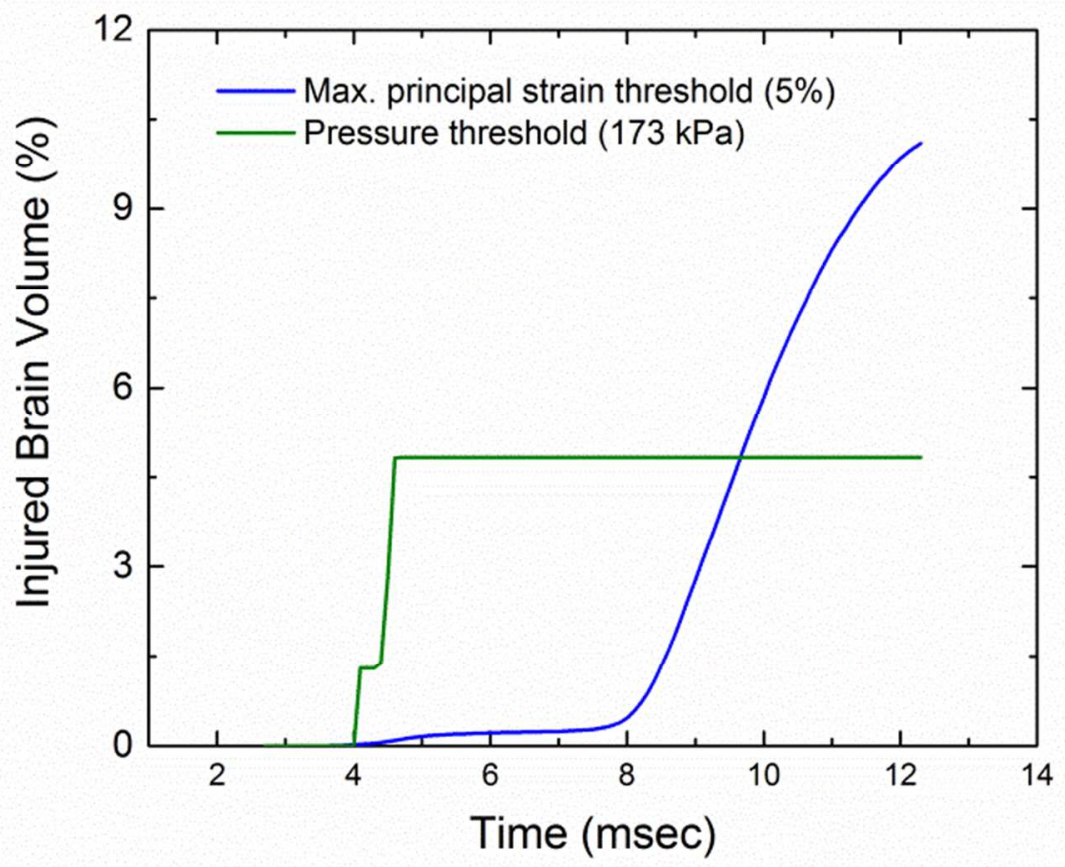

$170 \times 130 \mathrm{~mm}(120 \times 120 \mathrm{DPI})$ 


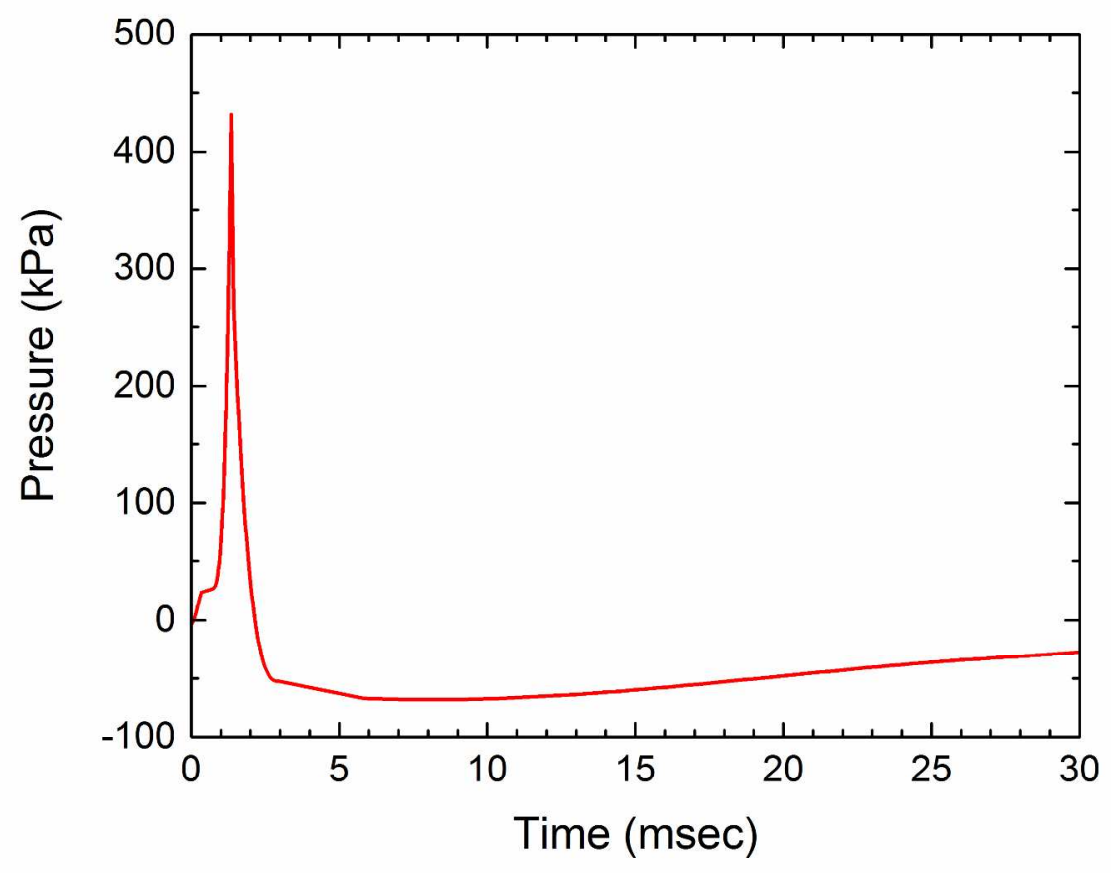

$271 \times 203 \mathrm{~mm}(300 \times 300 \mathrm{DPI})$

URL: http://mc.manuscriptcentral.com/gcmb 


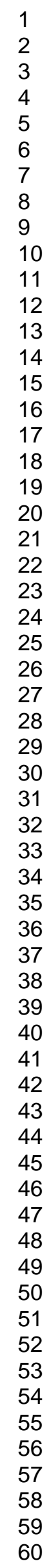

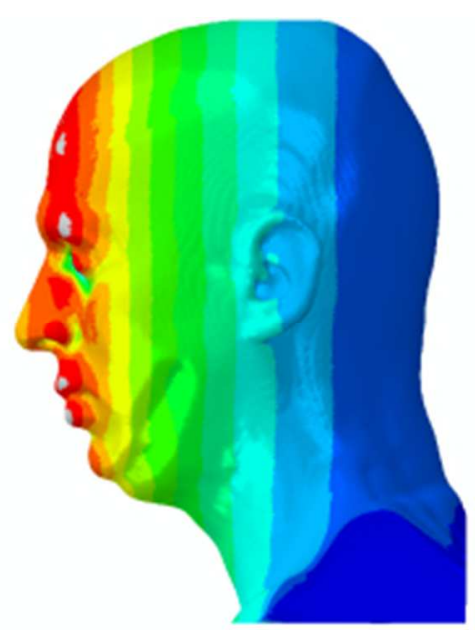

$22 \times 27 \mathrm{~mm}(220 \times 220 \mathrm{DPI})$

29

30 


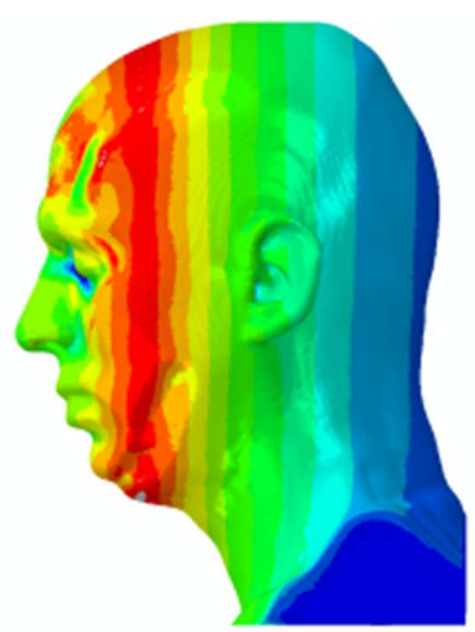

$22 \times 27 \mathrm{~mm}(220 \times 220 \mathrm{DPI})$ 


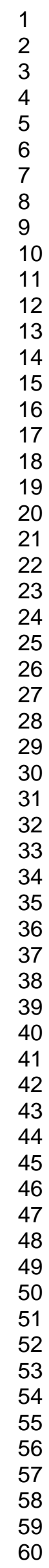

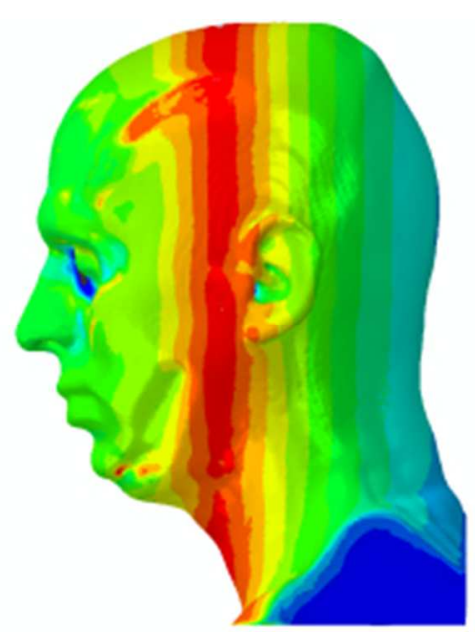

$22 \times 27 \mathrm{~mm}(220 \times 220 \mathrm{DPI})$ 6 


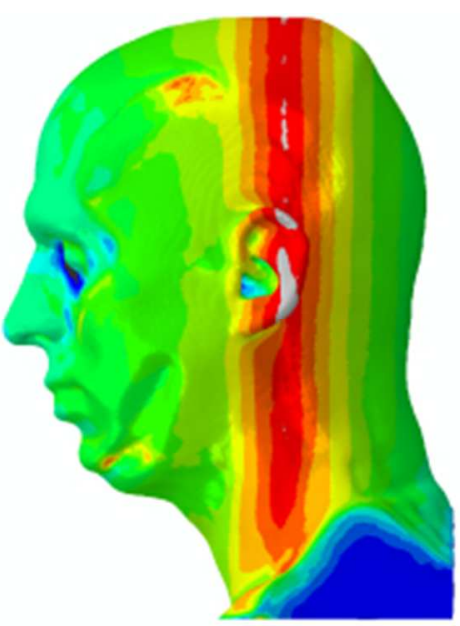

$22 \times 27 m m(220 \times 220$ DPI $)$ 


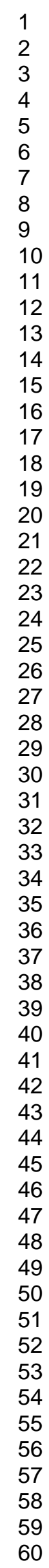

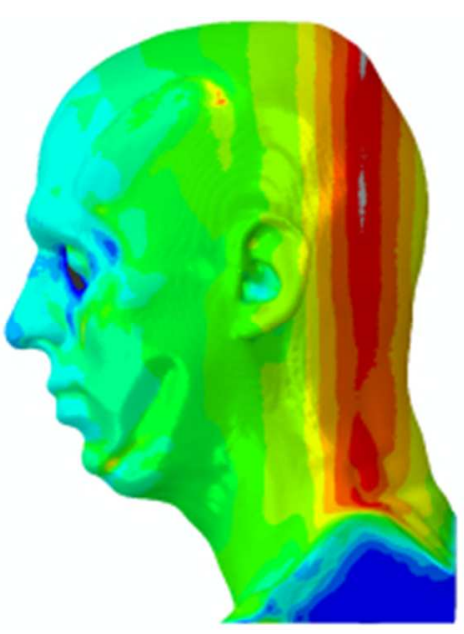

$22 \times 27 \mathrm{~mm}(220 \times 220 \mathrm{DPI})$

URL: http://mc.manuscriptcentral.com/gcmb 


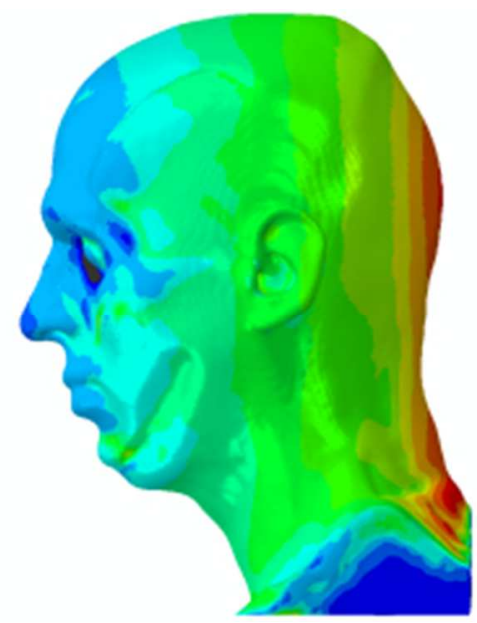

$22 \times 27 \mathrm{~mm}(220 \times 220$ DPI $)$ 


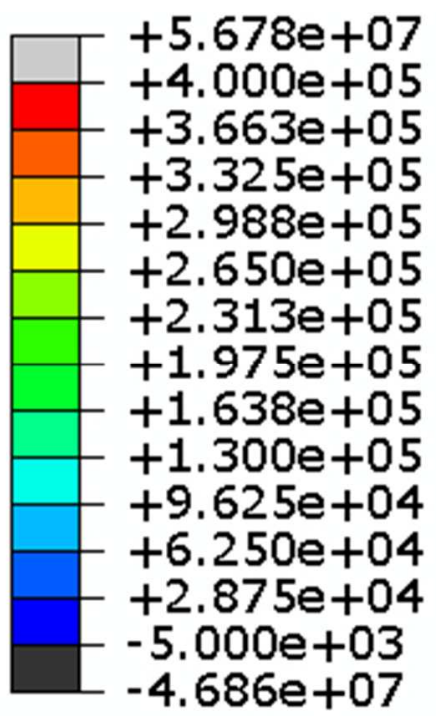

$61 \times 95 \mathrm{~mm}(72 \times 72 \mathrm{DPI})$ 


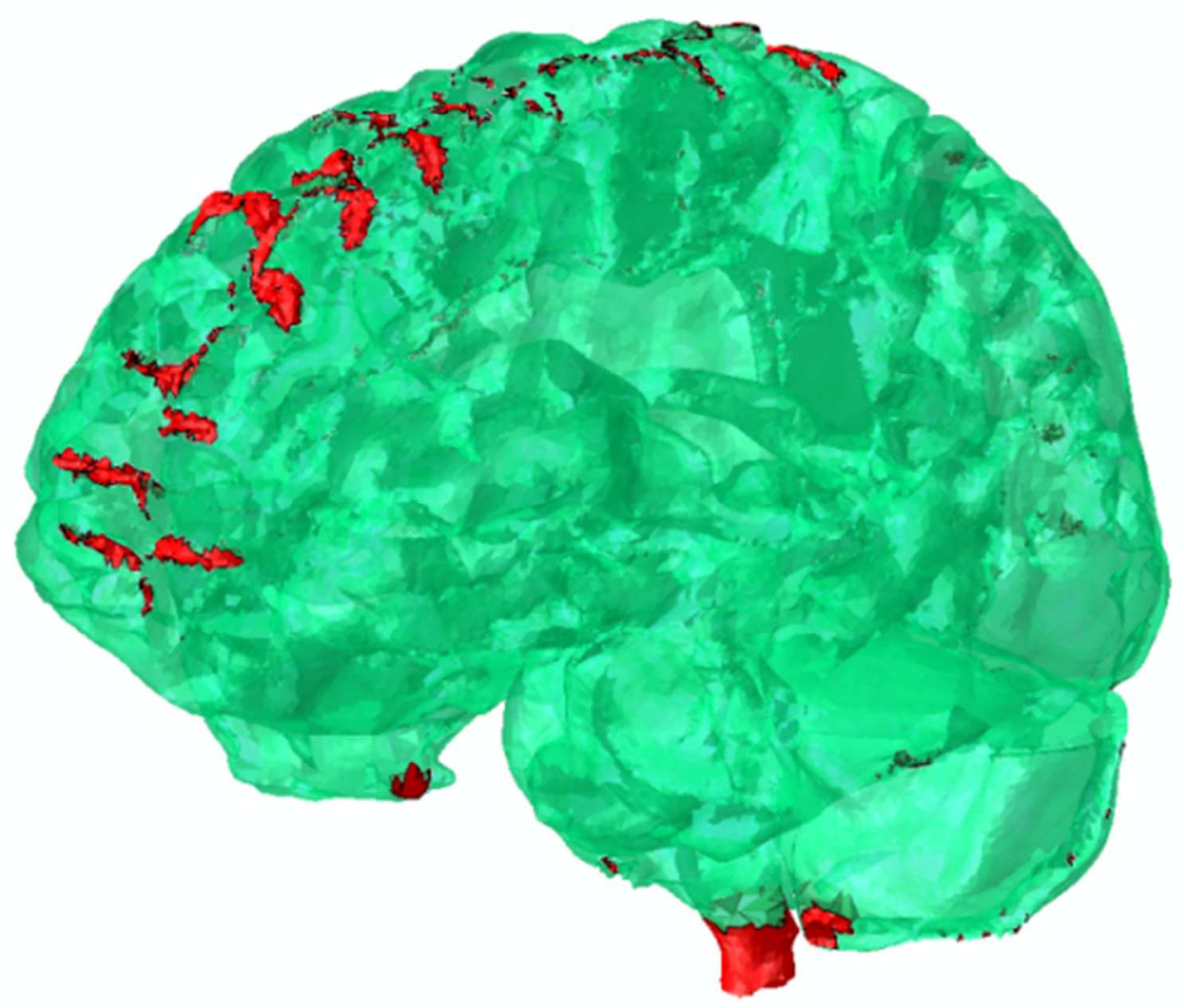

$68 \times 56 \mathrm{~mm}(220 \times 220 \mathrm{DPI})$ 


1
2
3
4
5
6
7
8
9
10
11
12
13
14
15
16
17
18
19
20
21
22
23
24
25
26
27
28
29
30
31
32
33
34
35
36
37
38
39
40
41
42
43
44
45
46
47
50
58
59
50
52
53
50

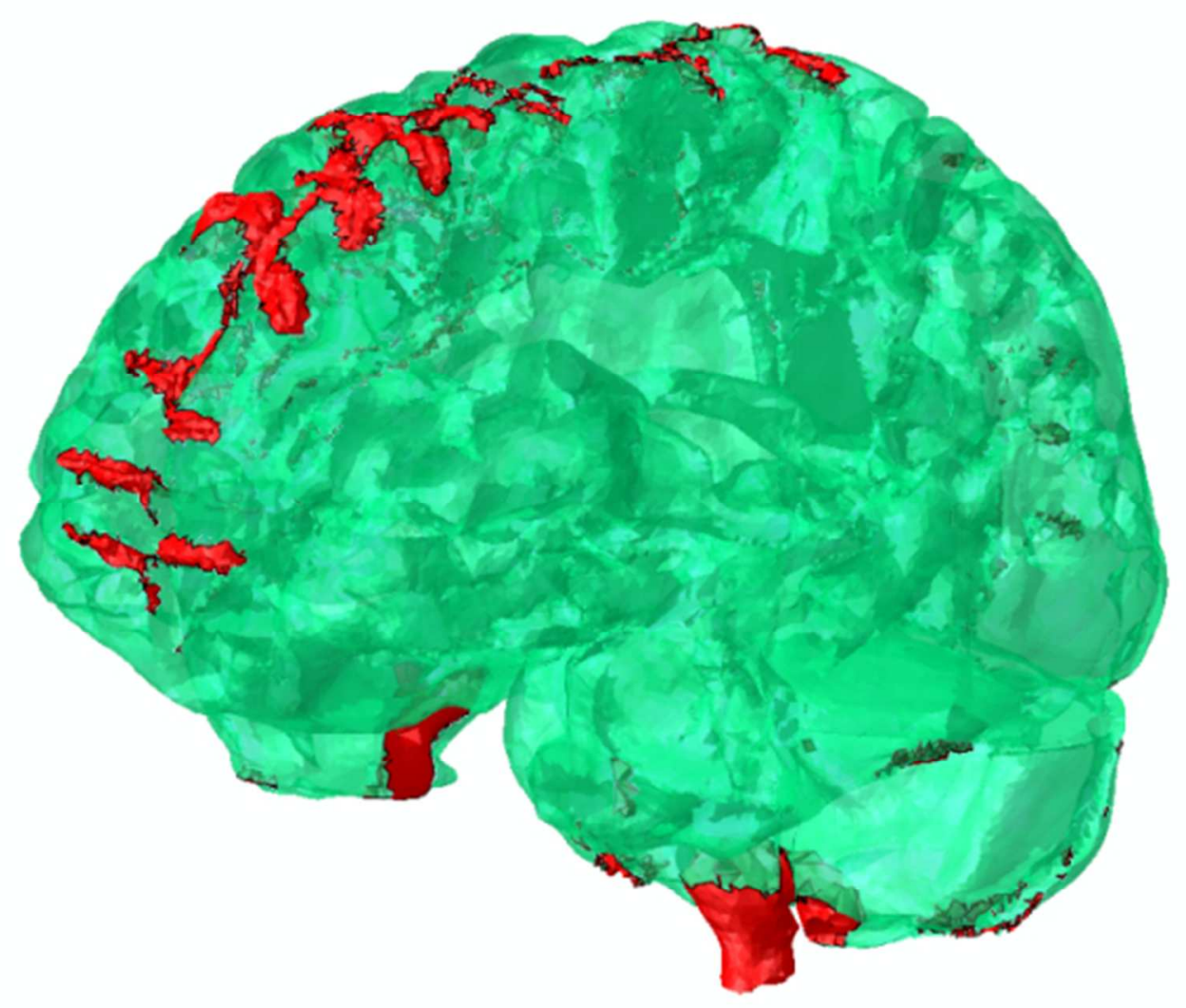

$68 \times 56 \mathrm{~mm}(220 \times 220$ DPI $)$ 